\title{
A New Strategy for the Synthesis of 1,4-Benzodiazepine Derivatives Based on the Tandem $N$-Alkylation-Ring Opening-Cyclization Reactions of Methyl 1-Arylaziridine-2-carboxylates with $N$-[2-Bromomethyl(phenyl)]- trifluoroacetamides
}

Jin-Yuan Wang, Xue-Fei Guo, De-Xian Wang, Zhi-Tang Huang, Mei-Xiang Wang*

Beijing National Laboratory for Molecular Sciences, Laboratory of Chemical Biology, Institute of Chemistry, Chinese Academy of Sciences, Beijing 100080, China

\section{mxwang@iccas.ac.cn}

\section{Table of Contents}

1. General experimental methods and spectroscopic data of compounds prepared

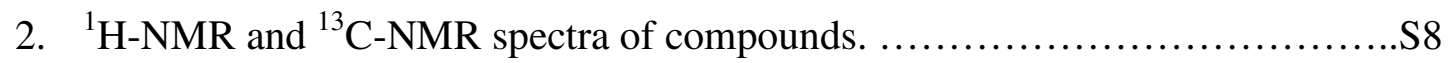

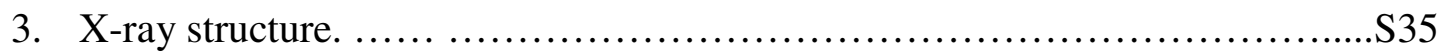

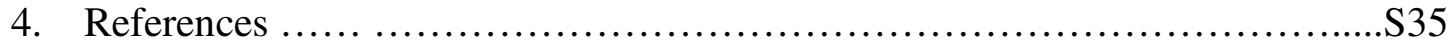

1. General experimental methods and spectroscopic data of compounds prepared. 
Both melting points and boiling points are uncorrected. The substrates 1a, 1g-j and 2a-f were prepared following the literature methods. ${ }^{1,2}$

N-(2-(Bromomethyl)-3-chlorophenyl)-2,2,2-trifluoroacetamide 11e: mp $154-155^{\circ} \mathrm{C} ;{ }^{1} \mathrm{H}$ NMR $\left(300 \mathrm{MHz}, \mathrm{CDCl}_{3}, \mathrm{TMS}\right) \delta 8.30(\mathrm{br}, 1 \mathrm{H}), 7.79-7.76(\mathrm{~m}, 1 \mathrm{H})$, 7.37-7.35(m, 2H), 4.67(m, 2H); ${ }^{13} \mathrm{C}$ NMR (75MHz, $\mathrm{CDCl}_{3}$, TMS) $\delta 155.4(\mathrm{q}, J=37.7$ $\mathrm{Hz}), 135.0,134.8,130.4,128.4,128.0,123.4,115.7(\mathrm{q}, J=286.8 \mathrm{~Hz}), 25.6$; IR (KBr) $v$ 3265(NH), 1638(C=O) cm $\mathrm{cm}^{-1}$; MS (EI) m/z (\%) $315(5)\left[\mathrm{M}^{+}\right], 317(7)\left[\mathrm{M}^{+}+2\right], 319$ (2) [M+4], 238 (20), 236 (100); Anal. Calcd for: $\mathrm{C}_{9} \mathrm{H}_{6} \mathrm{~F}_{3} \mathrm{ClBrNO}$;, $34.15 ; \mathrm{H}, 1.91$; N, 4.43; Found: C, 34.37; H, 1.99; N, 4.13.

Ring-opening Reaction of Methyl 1-Phenylaziridine-2-carboxylate 12a with $N$-(2-(bromomethyl)phenyl)-2,2,2-trifluoroacetamide 11a.

A mixture of methyl 1-phenylaziridine-2-carboxylate 12a $(1 \mathrm{mmol})$ and $\mathrm{N}$-(2-(bromomethyl)phenyl)-2,2,2-trifluoroacetamide 11a (1 mmol) in acetonitrile (10 $\mathrm{mL}$ ) was refluxed for 11 hours. After completion of the reaction, which was monitored by TLC, the reaction mixture was cooled to room temperature and the solvent was removed under vacuum. The residue was chromatographed on a silica gel column eluting with a mixture of petroleum ether and ethyl acetate to give a mixture of 13a and 14a. Products 13a and 14a were not separable by column chromatography. Their ratio was determined roughly by ${ }^{1} \mathrm{H}$ NMR spectrum.

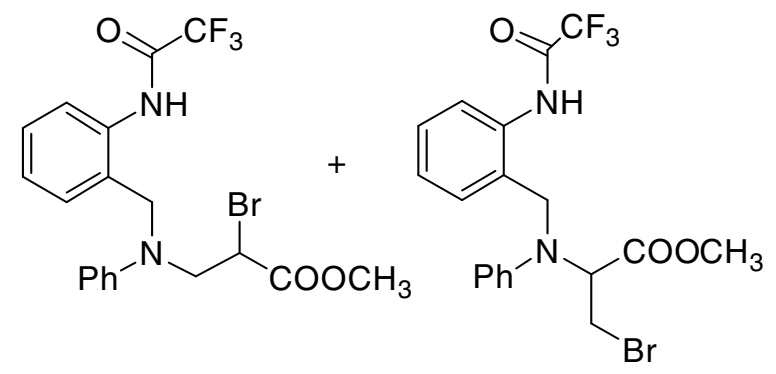

13a + 14a: oil; 13a ${ }^{1} \mathrm{H}$ NMR (300MHz, $\mathrm{CDCl}_{3}$, TMS) $\delta 10.0$ (br, 1H), 8.11-8.07 (m, 1H), 7.41-7.28 (m, 3H), 7.24-7.18 (m, 2H), 7.13-7.06 (m, 3H), 4.41 (s, 2H), 4.22 (dd, $J$ $=5.9 \mathrm{~Hz}, 8.5 \mathrm{~Hz}, 1 \mathrm{H}), 3.83(\mathrm{dd}, J=8.5 \mathrm{~Hz}, 14.4 \mathrm{~Hz}, 1 \mathrm{H}), 3.61(\mathrm{~s}, 3 \mathrm{H}), 3.54(\mathrm{dd}, J=$ $6.0 \mathrm{~Hz}, 14.4 \mathrm{~Hz}, 1 \mathrm{H}) ; \mathbf{1 3 a}{ }^{13} \mathrm{C} \mathrm{NMR}\left(75 \mathrm{MHz}, \mathrm{CDCl}_{3}, \mathrm{TMS}\right) \delta 168.3,154.0$ (q, $J=36.5$ Hz), 146.3, 134.1, 129.1, 128.7, 128.2, 126.2, 125.1, 122.5, 121.8, 118.8, 114.9 (q, $J=$ $268.4 \mathrm{~Hz}), 55.4,54.9,52.0,39.4 ; 14 a{ }^{1} \mathrm{H}$ NMR (300MHz, $\left.\mathrm{CDCl}_{3}, \mathrm{TMS}\right) \delta 10.0(\mathrm{br}$, 
0.1H), 8.11-8.07 (m, 0.1H), 7.41-7.28 (m, 0.3H), 7.24-7.18 (m, 0.2H), 7.13-7.06 (m, $0.3 \mathrm{H}), 4.65(\mathrm{~d}, J=13.7 \mathrm{~Hz}, 0.1 \mathrm{H}), 4.15(\mathrm{dd}, J=5.8 \mathrm{~Hz}, 8.2 \mathrm{~Hz}, 0.1 \mathrm{H}), 3.70(\mathrm{dd}, J=5.9$ $\mathrm{Hz}, 10.9 \mathrm{~Hz}, 0.1 \mathrm{H}), 3.71$ (s, 0.3H), 3.51 (dd, $J=8.2 \mathrm{~Hz}, 10.9 \mathrm{~Hz}, 0.1 \mathrm{H}) ; 14 \mathbf{a}^{13} \mathrm{C} \mathrm{NMR}$ $\left(75 \mathrm{MHz}, \mathrm{CDCl}_{3}, \mathrm{TMS}\right) \delta 168.9,154.0(\mathrm{q}, J=36.5 \mathrm{~Hz}), 145.0,134.6,130.1,128.4$, 128.1, 126.0, 125.3, 123.7, 121.7, 121.2, 114.9 (q, $J=268.4$ Hz), 64.0, 53.4, 51.4, 27.7; IR $(\mathrm{KBr}) \vee 3277(\mathrm{NH}), 1734(\mathrm{C}=\mathrm{O}) \mathrm{cm}^{-1} ; \mathrm{MS}(\mathrm{ESI}) \mathrm{m} / \mathrm{z}(\%) 458.8(96)\left[\mathrm{M}+\mathrm{H}^{+}\right], 460.7$ (100) $\left[\mathrm{M}+2+\mathrm{H}^{+}\right]$; HRMS Calcd for: $\mathrm{C}_{19} \mathrm{H}_{18} \mathrm{~F}_{3} \mathrm{BrN}_{2} \mathrm{O}_{3}$; $\mathrm{Ms}, 458.0453\left[\mathrm{M}^{+}\right], 460.0432$ [M+2]; Found: C, Ms, $458.0459\left[\mathrm{M}^{+}\right], 460.0437\left[\mathrm{M}^{+}+2\right]$.

Intramolecular Cyclization Reaction of 13a+14a: A mixture of 13a+14a $(0.5 \mathrm{mmol})$ and base $(0.6 \mathrm{mmol})$ in acetonitrile $(10 \mathrm{~mL})$ was refluxed for 12 hours until the starting $\mathbf{1 3 a} \mathbf{a} \mathbf{1 4 a}$ was consumed, which was monitored by TLC. After cooling to room temperature, the solvent was removed under vacuum, and water was added and extracted with ether. Organic phase was dried with anhydrous $\mathrm{MgSO}_{4}$, and the solvent was removed under vacuum. The residue was chromatographed on a silica gel column eluting with a mixture of petroleum ether and ethyl acetate to give pure $\mathbf{1 5 a}$.

One-pot Reaction of 11 and 12 to Prepare 1, 4-Benzodiazepine 15a-j. A mixture of $11(1 \mathrm{mmol})$ and $12(1 \mathrm{mmol})$ in acetonitrile $(10 \mathrm{~mL})$ was refluxed for 11 hours until the starting materials $\mathbf{1 1}$ and $\mathbf{1 2}$ were consumed, which was monitored by TLC. After cooling to room temperature, triethylamine $(1.2 \mathrm{mmol})$ was added, and the resulting mixture was refluxed for 12 hours. The solvent was removed under vacuum, and water was added and extracted with ether. Organic phase was dried with anhydrous $\mathrm{MgSO}_{4}$, and the solvent was removed under vacuum. The residue was chromatographed on a silica gel column eluting with a mixture of petroleum ether and ethyl acetate to give pure 15. 


\section{Methyl}

4-phenyl-1-(2,2,2-trifluoroacetyl)-2,3,4,5-tetrahydro-1H-benzo[e][1,4]diazepine-2 -carboxylate 15a: $\mathrm{mp} 129-130{ }^{\circ} \mathrm{C} ;{ }^{1} \mathrm{H}$ NMR $\left(300 \mathrm{MHz}, \mathrm{CDCl}_{3}\right.$, TMS) $\delta$ 7.51-7.44 (m, 4H), 7.41-7.25 (m, 2H), 6.88-6.81 (m, 3H), $5.35(\mathrm{dd}, J=4.5 \mathrm{~Hz}, 11.1 \mathrm{~Hz}, 1 \mathrm{H}), 4.53(\mathrm{~d}$, $J=12.7 \mathrm{~Hz}, 1 \mathrm{H}), 4.45(\mathrm{~d}, J=12.7 \mathrm{~Hz}, 1 \mathrm{H}), 4.19(\mathrm{dd}, J=4.5 \mathrm{~Hz}, 15.9 \mathrm{~Hz}, 1 \mathrm{H}), 3.76(\mathrm{~s}$,

3H), $3.27(\mathrm{dd}, J=11.1 \mathrm{~Hz}, 15.9 \mathrm{~Hz}, 1 \mathrm{H}) ;{ }^{13} \mathrm{C} \mathrm{NMR}\left(75 \mathrm{MHz}, \mathrm{CDCl}_{3}\right.$, TMS) $\delta 168.4$, $157.8(\mathrm{q}, J=36.6 \mathrm{~Hz}), 147.1,134.8,133.9,130.3,130.1,129.7,129.6,129.3,118.2$, $114.9(\mathrm{q}, J=286.8 \mathrm{~Hz}), 112.4,57.4,53.0,52.6,44.7$; IR $(\mathrm{KBr}) \vee 1639(\mathrm{C}=\mathrm{O}) \mathrm{cm}^{-1}$; MS (ESI) $\mathrm{m} / \mathrm{z} \quad(\%) \quad 378.9$ (100) $\quad\left[\mathrm{M}+\mathrm{H}^{+}\right], 401.0$ (40) $\left[\mathrm{M}+\mathrm{Na}^{+}\right]$; Anal. Calcd for: $\mathrm{C}_{19} \mathrm{H}_{17} \mathrm{~F}_{3} \mathrm{~N}_{2} \mathrm{O}_{3} ; \mathrm{C}, 60.32 ; \mathrm{H}, 4.53 ; \mathrm{N}, 7.40$; Found: C, 60.36; H, 4.69; N, 7.49.

\section{Methyl}

4-(4-fluorophenyl)-1-(2,2,2-trifluoroacetyl)-2,3,4,5-tetrahydro-1H-benzo[e][1,4]di azepine-2-carboxylate 15b: mp $124-125^{\circ} \mathrm{C} ;{ }^{1} \mathrm{H}$ NMR $\left(300 \mathrm{MHz}, \mathrm{CDCl}_{3}, \mathrm{TMS}\right) \delta$ 7.53-7.43 (m, 4H), 7.05-6.99 (t, $J=9.0 \mathrm{~Hz}, 2 \mathrm{H}), 6.82-6.77(\mathrm{~m}, 2 \mathrm{H}), 5.35(\mathrm{dd}, J=4.5$ $\mathrm{Hz}, 10.9 \mathrm{~Hz}, 1 \mathrm{H}), 4.68(\mathrm{~d}, J=12.5 \mathrm{~Hz}, 1 \mathrm{H}), 4.40(\mathrm{~d}, J=12.5 \mathrm{~Hz}, 1 \mathrm{H}), 4.08$ (dd, $J=4.5$ $\mathrm{Hz}, 15.9 \mathrm{~Hz}, 1 \mathrm{H}), 3.75(\mathrm{~s}, 3 \mathrm{H}), 3.27(\mathrm{dd}, J=10.9 \mathrm{~Hz}, 15.9 \mathrm{~Hz}, 1 \mathrm{H}) ;{ }^{13} \mathrm{C}$ NMR $(75 \mathrm{MHz}$, $\left.\mathrm{CDCl}_{3}, \mathrm{TMS}\right) \delta 168.3,157.8(\mathrm{q}, J=36.5 \mathrm{~Hz}), 157.7,154.6,143.8,134.8,133.8,130.3$, 130.1, 129.4, 129.3, 116.3, 116.0, 115.8, $114.9(\mathrm{q}, J=286.8 \mathrm{~Hz}), 113.7,113.6,57.2$, 53.4, 52.6, 45.4; IR (KBr) v 1755, 1708, $1639(\mathrm{C}=\mathrm{O}) \mathrm{cm}^{-1}$; MS (ESI) m/z (\%) 397.1 (40) $\left[\mathrm{M}+\mathrm{H}^{+}\right], 419.1(100)\left[\mathrm{M}+\mathrm{Na}^{+}\right]$; Anal. Calcd for: $\mathrm{C}_{19} \mathrm{H}_{16} \mathrm{~F}_{4} \mathrm{~N}_{2} \mathrm{O}_{3} ; \mathrm{C}, 57.58 ; \mathrm{H}, 4.07$; N, 7.07; Found: C, 57.65; H, 4.11; N, 7.15.

\section{Methyl}

4-(4-chlorophenyl)-1-(2,2,2-trifluoroacetyl)-2,3,4,5-tetrahydro-1H-benzo[e][1,4]di azepine-2-carboxylate 15c: $\mathrm{mp} 112-113^{\circ} \mathrm{C} ;{ }^{1} \mathrm{H}$ NMR $\left(300 \mathrm{MHz}, \mathrm{CDCl}_{3}, \mathrm{TMS}\right) \delta$ 7.51-7.40 (m, 4H), 7.27-7.23 (m, 2H), 6.79-6.76 (m, 2H), 5.35 (dd, J = 4.5 Hz, $11.0 \mathrm{~Hz}$, 1H), $4.49(\mathrm{~d}, J=12.7 \mathrm{~Hz}, 1 \mathrm{H}), 4.40(\mathrm{~d}, J=12.7 \mathrm{~Hz}, 1 \mathrm{H}), 4.11(\mathrm{dd}, J=4.5 \mathrm{~Hz}, 15.9 \mathrm{~Hz}$, 1H), $3.76(\mathrm{~s}, 3 \mathrm{H}), 3.27(\mathrm{dd}, J=11.0 \mathrm{~Hz}, 15.9 \mathrm{~Hz}, 1 \mathrm{H}) ;{ }^{13} \mathrm{C} \mathrm{NMR}\left(75 \mathrm{MHz}, \mathrm{CDCl}_{3}\right.$, TMS) $\delta 168.2,157.8(\mathrm{q}, J=36.5 \mathrm{~Hz}), 145.7,134.7,133.6,130.3,130.2,129.6,129.5$, $129.4,123.1,116.3$ (q, $J=286.9 \mathrm{~Hz}), 113.5,57.1,53.2,52.7,44.8 ; \mathrm{IR}(\mathrm{KBr}) \vee 1638$ $(\mathrm{C}=\mathrm{O}) \mathrm{cm}^{-1}$; MS (ESI) m/z (\%) $413.1(100)\left[\mathrm{M}+\mathrm{H}^{+}\right], 415.2(40)\left[\mathrm{M}+2+\mathrm{H}^{+}\right]$; Anal 
Calcd for: $\mathrm{C}_{19} \mathrm{H}_{16} \mathrm{ClF}_{3} \mathrm{~N}_{2} \mathrm{O}_{3} ; \mathrm{C}, 55.28 ; \mathrm{H}, 3.91 ; \mathrm{N}, 6.79$; Found: C, 55.32; H, 3.91; N, 6.71 .

\section{Methyl}

4-p-tolyl-1-(2,2,2-trifluoroacetyl)-2,3,4,5-tetrahydro-1H-benzo[e][1,4]diazepine-2carboxylate 15d: $\mathrm{mp} 161-162^{\circ} \mathrm{C} ;{ }^{1} \mathrm{H} \mathrm{NMR}\left(300 \mathrm{MHz}, \mathrm{CDCl}_{3}\right.$, TMS) $\delta$ 7.50-7.41 (m, 4H), 7.26-7.12 (m, 2H), 6.80-6.77 (m, 2H), $5.34(\mathrm{dd}, J=4.5 \mathrm{~Hz}, 11.0 \mathrm{~Hz}, 1 \mathrm{H}), 4.50(\mathrm{~d}$, $J=12.5 \mathrm{~Hz}, 1 \mathrm{H}), 4.43(\mathrm{~d}, J=12.5 \mathrm{~Hz}, 1 \mathrm{H}), 4.14(\mathrm{dd}, J=4.5 \mathrm{~Hz}, 15.9 \mathrm{~Hz}, 1 \mathrm{H}), 3.76(\mathrm{~s}$,

$3 \mathrm{H}), 3.24(\mathrm{dd}, J=11.0 \mathrm{~Hz}, 15.9 \mathrm{~Hz}, 1 \mathrm{H}), 2.29(\mathrm{~s}, 3 \mathrm{H}) ;{ }^{13} \mathrm{C} \mathrm{NMR}\left(75 \mathrm{MHz}, \mathrm{CDCl}_{3}\right.$, TMS) $\delta 168.2,157.8(\mathrm{q}, J=36.5 \mathrm{~Hz}), 168.5,157.8(\mathrm{q}, J=36.6 \mathrm{~Hz}), 145.1,134.9$, 134.0, 130.2, 130.0, 129.8, 129.6, 129.2, 127.5, 116.3 (q, $J=286.8 \mathrm{~Hz}), 112.7,57.3$, 53.2, 52.6, 45.0, 20.2; IR (KBr) $\vee 1638(\mathrm{C}=\mathrm{O}) \mathrm{cm}^{-1}$; MS (ESI) m/z (\%) 393.1 (24) $\left[\mathrm{M}+\mathrm{H}^{+}\right], 415.2(100)\left[\mathrm{M}+\mathrm{Na}^{+}\right]$; Anal. Calcd for: $\mathrm{C}_{20} \mathrm{H}_{19} \mathrm{~F}_{3} \mathrm{~N}_{2} \mathrm{O}_{3} ; \mathrm{C}, 61.22 ; \mathrm{H}, 4.88 ; \mathrm{N}$, 7.14; Found: C, 61.21; H, 4.74; N, 7.05.

\section{Methyl}

4-m-Tolyl-1-(2,2,2-trifluoroacetyl)-2,3,4,5-tetrahydro-1H-benzo[e][1,4]diazepine2-carboxylate 15e: $\mathrm{mp} 123-124^{\circ} \mathrm{C} ;{ }^{1} \mathrm{H}$ NMR $\left(300 \mathrm{MHz}, \mathrm{CDCl}_{3}\right.$, TMS) $\delta$ 7.52-7.41 (m, 4H), 7.23-7.18 (m, 1H), 6.69-6.65 (m, 3H), $5.36(\mathrm{dd}, J=4.5 \mathrm{~Hz}, 11.1 \mathrm{~Hz}, 1 \mathrm{H}), 4.54(\mathrm{~d}$, $J=12.7 \mathrm{~Hz}, 1 \mathrm{H}), 4.45(\mathrm{~d}, J=12.7 \mathrm{~Hz}, 1 \mathrm{H}), 4.18(\mathrm{dd}, J=4.5 \mathrm{~Hz}, 15.9 \mathrm{~Hz}, 1 \mathrm{H}), 3.77(\mathrm{~s}$, $3 \mathrm{H}), 3.25(\mathrm{dd}, J=11.1 \mathrm{~Hz}, 15.9 \mathrm{~Hz}, 1 \mathrm{H}), 2.36(\mathrm{~s}, 3 \mathrm{H}) ;{ }^{13} \mathrm{C} \mathrm{NMR}\left(75 \mathrm{MHz}, \mathrm{CDCl}_{3}\right.$, TMS) $\delta 168.4,157.8(\mathrm{q}, J=36.7 \mathrm{~Hz}), 147.2,139.5,134.8,134.0,130.3,130.0,129.6$, 129.5, 129.2, 119.2, 116.0 (q, $J=286.8 \mathrm{~Hz}), 113.2$, 109.6, 57.4, 53.1, 52.6, 44.7, 22.0; IR $(\mathrm{KBr}) \vee 1638(\mathrm{C}=\mathrm{O}) \mathrm{cm}^{-1}$; MS (ESI) m/z (\%) $393.0(100)\left[\mathrm{M}+\mathrm{H}^{+}\right]$; Anal. Calcd for: $\mathrm{C}_{20} \mathrm{H}_{19} \mathrm{~F}_{3} \mathrm{~N}_{2} \mathrm{O}_{3} ; \mathrm{C}, 61.22 ; \mathrm{H}, 4.88 ; \mathrm{N}, 7.14$; Found: $\mathrm{C}, 61.14 ; \mathrm{H}, 4.91 ; \mathrm{N}, 7.11$.

\section{Methyl}

\section{4-(4-methoxyphenyl)-1-(2,2,2-trifluoroacetyl)-2,3,4,5-tetrahydro-1H-benzo[e][1,4}

]diazepine-2-carboxylate 15f: $\mathrm{mp} 161-162^{\circ} \mathrm{C}$; ${ }^{1} \mathrm{H} \mathrm{NMR}\left(300 \mathrm{MHz}, \mathrm{CDCl}_{3}\right.$, TMS $) \delta$ 7.52-7.42 (m, 4H), 6.92-6.79 (m, 4H), 5.33 (dd, $J=4.3 \mathrm{~Hz}, 10.8 \mathrm{~Hz}, 1 \mathrm{H},), 4.48$ (d, $J=$ $12.5 \mathrm{~Hz}, 1 \mathrm{H}), 4.40(\mathrm{~d}, J=12.5 \mathrm{~Hz}, 1 \mathrm{H}), 4.06(\mathrm{dd}, J=4.3 \mathrm{~Hz}, 15.8 \mathrm{~Hz}, 1 \mathrm{H}), 3.78(\mathrm{~s}$, $3 \mathrm{H}), 3.75(\mathrm{~s}, 3 \mathrm{H}), 3.24(\mathrm{dd}, J=10.8 \mathrm{~Hz}, 15.8 \mathrm{~Hz}, 1 \mathrm{H}) ;{ }^{13} \mathrm{C} \mathrm{NMR}\left(75 \mathrm{MHz}, \mathrm{CDCl}_{3}\right.$, TMS) $\delta 168.5,157.8(\mathrm{q}, J=36.3 \mathrm{~Hz}), 152.7,141.9,135.0,134.1,130.1,129.9,129.6$, 
$127.5,116.3(\mathrm{q}, J=287.0 \mathrm{~Hz}), 115.3,114.3,57.4,55.8,53.5,52.6,45.8$; IR $(\mathrm{KBr}) v$ $1639(\mathrm{C}=\mathrm{O}) \mathrm{cm}^{-1}$; MS (ESI) $\mathrm{m} / \mathrm{z}(\%) 409.0$ (100) $\left[\mathrm{M}+\mathrm{H}^{+}\right]$; Anal. Calcd for: $\mathrm{C}_{20} \mathrm{H}_{19} \mathrm{~F}_{3} \mathrm{~N}_{2} \mathrm{O}_{4} ; \mathrm{C}, 58.82 ; \mathrm{H}, 4.69 ; \mathrm{N}, 6.86$; Found: C, 58.65; H, 4.60; N, 6.61.

\section{Methyl}

\section{8-chloro-4-phenyl-1-(2,2,2-trifluoroacetyl)-2,3,4,5-tetrahydro-1H-benzo[e][1,4]di} azepine-2-carboxylate 15g: $\mathrm{mp} 138-139^{\circ} \mathrm{C} ;{ }^{1} \mathrm{H}$ NMR $\left(300 \mathrm{MHz}, \mathrm{CDCl}_{3}\right.$, TMS) $\delta$ 7.55-7.21 (m, 5H), 6.87-6.80 (m, 3H), $5.35(\mathrm{dd}, J=4.5 \mathrm{~Hz}, 11.0 \mathrm{~Hz}, 1 \mathrm{H}), 4.49$ (d, $J=$ 13.2 Hz, 1H), $4.44(\mathrm{~d}, J=13.2 \mathrm{~Hz}, 1 \mathrm{H}), 4.20(\mathrm{dd}, J=4.5 \mathrm{~Hz}, 16.0 \mathrm{~Hz}, 1 \mathrm{H}), 3.78$ (s, $3 \mathrm{H}), 3.26(\mathrm{dd}, J=11.0 \mathrm{~Hz}, 16.0 \mathrm{~Hz}, 1 \mathrm{H}) ;{ }^{13} \mathrm{C} \mathrm{NMR}\left(75 \mathrm{MHz}, \mathrm{CDCl}_{3}, \mathrm{TMS}\right) \delta 168.1$, $157.6(q, J=36.8 \mathrm{~Hz}), 147.0,135.8,134.8,132.6$, 130.6, 130.5, 130.1, 129.8, 118.5, $115.3(\mathrm{q}, J=286.6 \mathrm{~Hz}), 112.5,57.4,52.8,52.5,44.7$; IR $(\mathrm{KBr}) \vee 1639(\mathrm{C}=\mathrm{O}) \mathrm{cm}^{-1}$; MS (ESI) $\mathrm{m} / \mathrm{z}(\%) 413.1(100)\left[\mathrm{M}+\mathrm{H}^{+}\right], 415.2(30)\left[\mathrm{M}+2+\mathrm{H}^{+}\right]$; Anal. Calcd for: $\mathrm{C}_{19} \mathrm{H}_{16} \mathrm{ClF}_{3} \mathrm{~N}_{2} \mathrm{O}_{3} ; \mathrm{C}, 55.28 ; \mathrm{H}, 3.91 ; \mathrm{N}, 6.79$; Found: C, 58.19; H, 4.01; N, 6.54.

\section{Methyl}

7-chloro-4-phenyl-1-(2,2,2-trifluoroacetyl)-2,3,4,5-tetrahydro-1H-benzo[e][1,4]di azepine-2-carboxylate 15h: $\mathrm{mp} 107-108^{\circ} \mathrm{C} ;{ }^{1} \mathrm{H} \mathrm{NMR}\left(300 \mathrm{MHz}, \mathrm{CDCl}_{3}, \mathrm{TMS}\right) \delta$ 7.49-7.42 (m, 3H), 7.35-7.29 (m, 2H), 6.87-6.81 (m, 3H), 5.37 (dd, J=4.6 Hz, $10.9 \mathrm{~Hz}$, $1 \mathrm{H}), 4.52(\mathrm{~d}, J=13.0 \mathrm{~Hz}, 1 \mathrm{H}), 4.40(\mathrm{~d}, J=13.0 \mathrm{~Hz}, 1 \mathrm{H}), 4.18(\mathrm{dd}, J=4.6 \mathrm{~Hz}, 15.9 \mathrm{~Hz}$, 1H), $3.75(\mathrm{~s}, 3 \mathrm{H}), 3.28(\mathrm{dd}, J=10.9 \mathrm{~Hz}, 15.9 \mathrm{~Hz}, 1 \mathrm{H}) ;{ }^{13} \mathrm{C} \mathrm{NMR}\left(75 \mathrm{MHz}, \mathrm{CDCl}_{3}\right.$, TMS) $\delta 168.2,157.6$ (q, $J=36.7 \mathrm{~Hz}), 146.9,135.83,135.80,133.3,131.7,129.8$, $129.7,129.2,118.5,115.3(\mathrm{q}, J=286.2 \mathrm{~Hz}), 112.5,57.3,52.8,52.7,45.0$; IR $(\mathrm{KBr}) v$ $1639(\mathrm{C}=\mathrm{O}) \mathrm{cm}^{-1} ; \mathrm{MS}(\mathrm{ESI}) \mathrm{m} / \mathrm{z}(\%) 413.1(100)\left[\mathrm{M}+\mathrm{H}^{+}\right], 415.2(44)\left[\mathrm{M}+2+\mathrm{H}^{+}\right] ;$Anal. Calcd for: $\mathrm{C}_{19} \mathrm{H}_{16} \mathrm{ClF}_{3} \mathrm{~N}_{2} \mathrm{O}_{3} ; \mathrm{C}, 55.28 ; \mathrm{H}, 3.91$; N, 6.79; Found: C, 55.14; H, 3.93; N, 6.56.

\section{Methyl}

7-methyl-4-phenyl-1-(2,2,2-trifluoroacetyl)-2,3,4,5-tetrahydro-1H-benzo[e][1,4]di azepine-2-carboxylate 15i: mp $132-133^{\circ} \mathrm{C}$; ${ }^{1} \mathrm{H}$ NMR $\left(300 \mathrm{MHz}, \mathrm{CDCl}_{3}, \mathrm{TMS}\right) \delta$ 7.40-7.23 (m, 5H), 6.87-6.80 (m, 3H), $5.36(\mathrm{dd}, J=4.5 \mathrm{~Hz}, 11.1 \mathrm{~Hz}, 1 \mathrm{H}), 4.50(\mathrm{~d}, J=$ $12.6 \mathrm{~Hz}, 1 \mathrm{H}), 4.38(\mathrm{~d}, J=12.6 \mathrm{~Hz}, 1 \mathrm{H}), 4.17(\mathrm{dd}, J=4.5 \mathrm{~Hz}, 15.9 \mathrm{~Hz}, 1 \mathrm{H}), 3.75(\mathrm{~s}$, $3 \mathrm{H}), 3.26(\mathrm{dd}, J=11.1 \mathrm{~Hz}, 15.9 \mathrm{~Hz}, 1 \mathrm{H}), 2.42(\mathrm{~s}, 3 \mathrm{H}) ;{ }^{13} \mathrm{C} \mathrm{NMR}\left(75 \mathrm{MHz}, \mathrm{CDCl}_{3}\right.$, 
TMS) $\delta 168.5,157.9(\mathrm{q}, J=36.7 \mathrm{~Hz}), 147.2,140.3,133.6,132.1,130.4,130.2,130.0$, $129.7,118.1,115.0(\mathrm{q}, J=286.0 \mathrm{~Hz}), 112.3,57.3,53.0,52.6,44.7,21.2$; IR $(\mathrm{KBr}) v$ 1737, 1699, $1638(\mathrm{C}=\mathrm{O}) \mathrm{cm}^{-1}$; MS (ESI) m/z (\%) 393.1 (100) $\left[\mathrm{M}+\mathrm{H}^{+}\right]$; Anal. Calcd for: $\mathrm{C}_{20} \mathrm{H}_{19} \mathrm{~F}_{3} \mathrm{~N}_{2} \mathrm{O}_{3} ; \mathrm{C}, 61.22 ; \mathrm{H}, 4.88 ; \mathrm{N}, 7.14$; Found: C, 61.06; H, 4.98; N, 7.07.

\section{Methyl}

6-chloro-4-phenyl-1-(2,2,2-trifluoroacetyl)-2,3,4,5-tetrahydro-1H-benzo[e][1,4]di azepine-2-carboxylate 15j: mp $105-106^{\circ} \mathrm{C} ;{ }^{1} \mathrm{H}$ NMR $\left(300 \mathrm{MHz}, \mathrm{CDCl}_{3}, \mathrm{TMS}\right) \delta$ 7.56-7.25 (m, 5H), 6.93-6.84 (m, 3H), 5.35 (dd, $J=4.7 \mathrm{~Hz}, 10.9 \mathrm{~Hz}, 1 \mathrm{H}), 5.06$ (d, $J=$ $13.5 \mathrm{~Hz}, 1 \mathrm{H}), 4.37$ (d, $J=13.5 \mathrm{~Hz}, 1 \mathrm{H}), 4.19$ (dd, $J=4.7 \mathrm{~Hz}, 15.8 \mathrm{~Hz}, 1 \mathrm{H}), 3.76$ (s, $3 \mathrm{H}), 3.26(\mathrm{dd}, J=10.9 \mathrm{~Hz}, 15.8 \mathrm{~Hz}, 1 \mathrm{H}) ;{ }^{13} \mathrm{C} \mathrm{NMR}\left(75 \mathrm{MHz}, \mathrm{CDCl}_{3}, \mathrm{TMS}\right) \delta 168.3$, $157.6(\mathrm{q}, J=36.6 \mathrm{~Hz}), 147.3,136.5,134.2,132.2,131.3,129.7,129.5,129.3,118.8$, $115.6(\mathrm{q}, J=285.8 \mathrm{~Hz}), 113.3,57.6,52.7,49.3,45.4$; IR $(\mathrm{KBr}) \vee 1638(\mathrm{C}=\mathrm{O}) \mathrm{cm}^{-1}$; MS (ESI) $\mathrm{m} / \mathrm{z}(\%) 413.1$ (100) $\left[\mathrm{M}+\mathrm{H}^{+}\right], 415.2$ (40) $\left[\mathrm{M}+2+\mathrm{H}^{+}\right]$; Anal. Calcd for: $\mathrm{C}_{19} \mathrm{H}_{16} \mathrm{ClF}_{3} \mathrm{~N}_{2} \mathrm{O}_{3} ; \mathrm{C}, 55.28 ; \mathrm{H}, 3.91 ; \mathrm{N}, 6.79$; Found: C, 55.22; H, 3.88; N, 6.63.

Hydrolysis of Compound 15h. A solution of $\mathbf{1 5 h}(1 \mathrm{mmol})$ and $\mathrm{K}_{2} \mathrm{CO}_{3}(5 \mathrm{mmol})$ in a mixture of methanol and water $(\mathrm{v}: \mathrm{v}=5: 2,10 \mathrm{~mL})$ was stirred for 2 days until the starting materials $\mathbf{1 5 h}$ was consumed, which was monitored by TLC. After the removal of the solvent under vacuum, the residue was chromatographed on a reverse phase ODS column $(35-70 \mu \mathrm{m})$ eluting with a mixture of water and methanol to give pure $\mathbf{1 7}$ in $89 \%$ yield.

\section{7-Chloro-4-phenyl-2,3,4,5-tetrahydro-1H-benzo[e][1,4]diazepine-2-carboxylic} acid 17: $\mathrm{mp} 197-198^{\circ} \mathrm{C}{ }^{1} \mathrm{H}$ NMR $\left(300 \mathrm{MHz}, \mathrm{D}_{2} \mathrm{O}, \mathrm{TMS}\right) \delta$ 7.36-7.06 (m, 4H), 6.97-6.44 (m, 4H), $4.90(\mathrm{~d}, J=7.5 \mathrm{~Hz}, 1 \mathrm{H}), 4.23(\mathrm{~d}, J=16.5 \mathrm{~Hz}, 1 \mathrm{H}), 4.04-3.67(\mathrm{~m}, J$ 2H), $2.86(\mathrm{t}, J=12.4 \mathrm{~Hz}, 14.1 \mathrm{~Hz}, 1 \mathrm{H}) ;{ }^{13} \mathrm{C} \mathrm{NMR}\left(75 \mathrm{MHz}, \mathrm{D}_{2} \mathrm{O}, \mathrm{TMS}\right) \delta 170.6,149.6$, $138.8,136.5,134.5,133.6,132.0,131.8,131.4,121.1,115.6,60.0,55.0,51.6$; IR (KBr) v 3422 (br), $1636(\mathrm{C}=\mathrm{O}) \mathrm{cm}^{-1}$; MS (ESI) m/z (\%) 301.1 (100) [M-H'] 303.1 (30) $\left[\mathrm{M}+2-\mathrm{H}^{+}\right]$; Anal. Calcd for: $\mathrm{C}_{16} \mathrm{H}_{15} \mathrm{ClN}_{2} \mathrm{O}_{2} ; \mathrm{C}, 63.47 ; \mathrm{H}, 4.99 ; \mathrm{N}, 9.25$; Found: $\mathrm{C}$, 63.50; H, 4.98; N, 9.27. 
2. ${ }^{1} \mathrm{H}-\mathrm{NMR}$ and ${ }^{13} \mathrm{C}-\mathrm{NMR}$ spectra of $11 \mathrm{j}, 13 \mathrm{a}+14 \mathrm{a}, 15 \mathrm{a}-\mathrm{j}, 17$. 
$15 \mathrm{a}^{-1} \mathrm{H}$ NMR

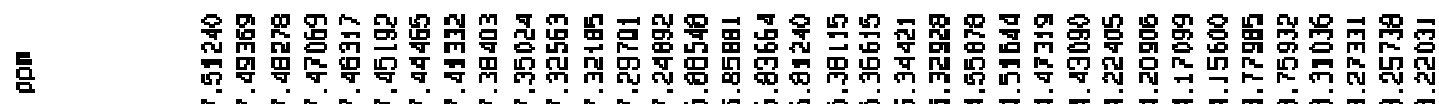

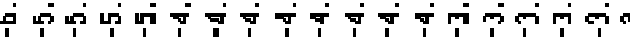

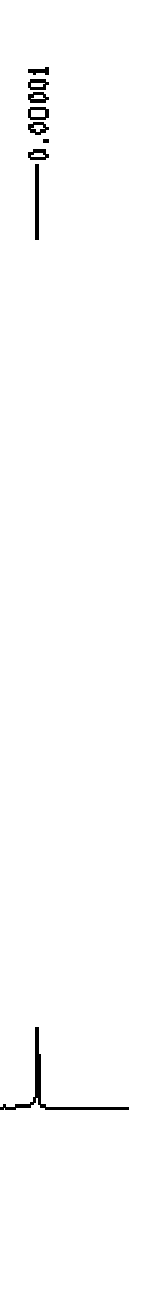

Cur rent Data Parsnaters

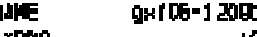

PFock

Fz - henusitulon Paroneters

Dite_ zopsisig

11n:

Jistill

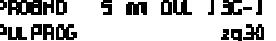

70. YE

DS

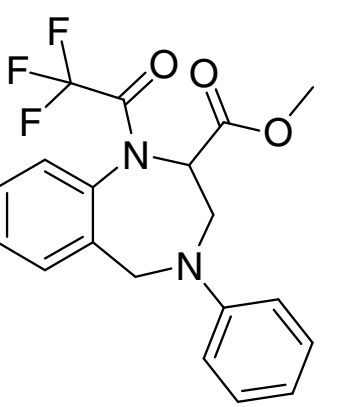

05

AD D.694JG Hz

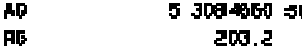

Df 2000.2

B.W ulet

$\begin{array}{ll}12 & 205.1 \mathrm{~K} \\ 01 & 2.0000000\end{array}$

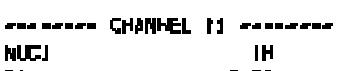

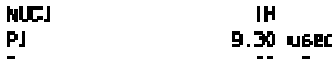

PLI $\quad-1.00100$

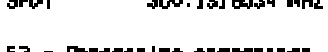

Fe - Procasting parsuecters

SF $301.1300093 \mathrm{mHz}$

MLI.

SEB
LE
GE

SE

D.

ID MHF p lat parametery
Co
CY

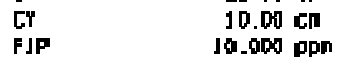

FI $361.30 \mathrm{~Hz}$

$\begin{array}{ll}\text { FPF } & -0.50 \mathrm{0ph} \\ \mathrm{FP} & -150.05 \mathrm{~Hz}\end{array}$

PPHCH $\quad 0.47727 \mathrm{gpul}$

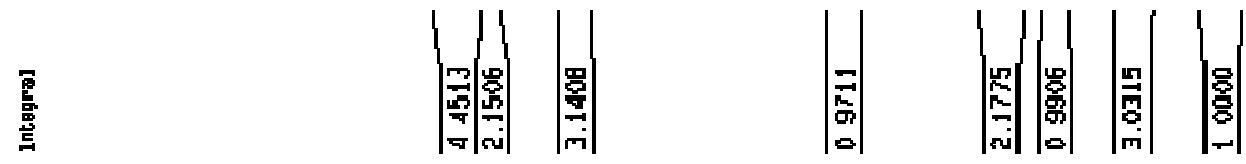

143. $240 \mathrm{H} \mathrm{HI}_{\mathrm{C}} \mathrm{CH}$

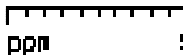

$g$ a

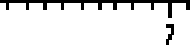


$15 \mathrm{a}-{ }^{13} \mathrm{C} N M R$

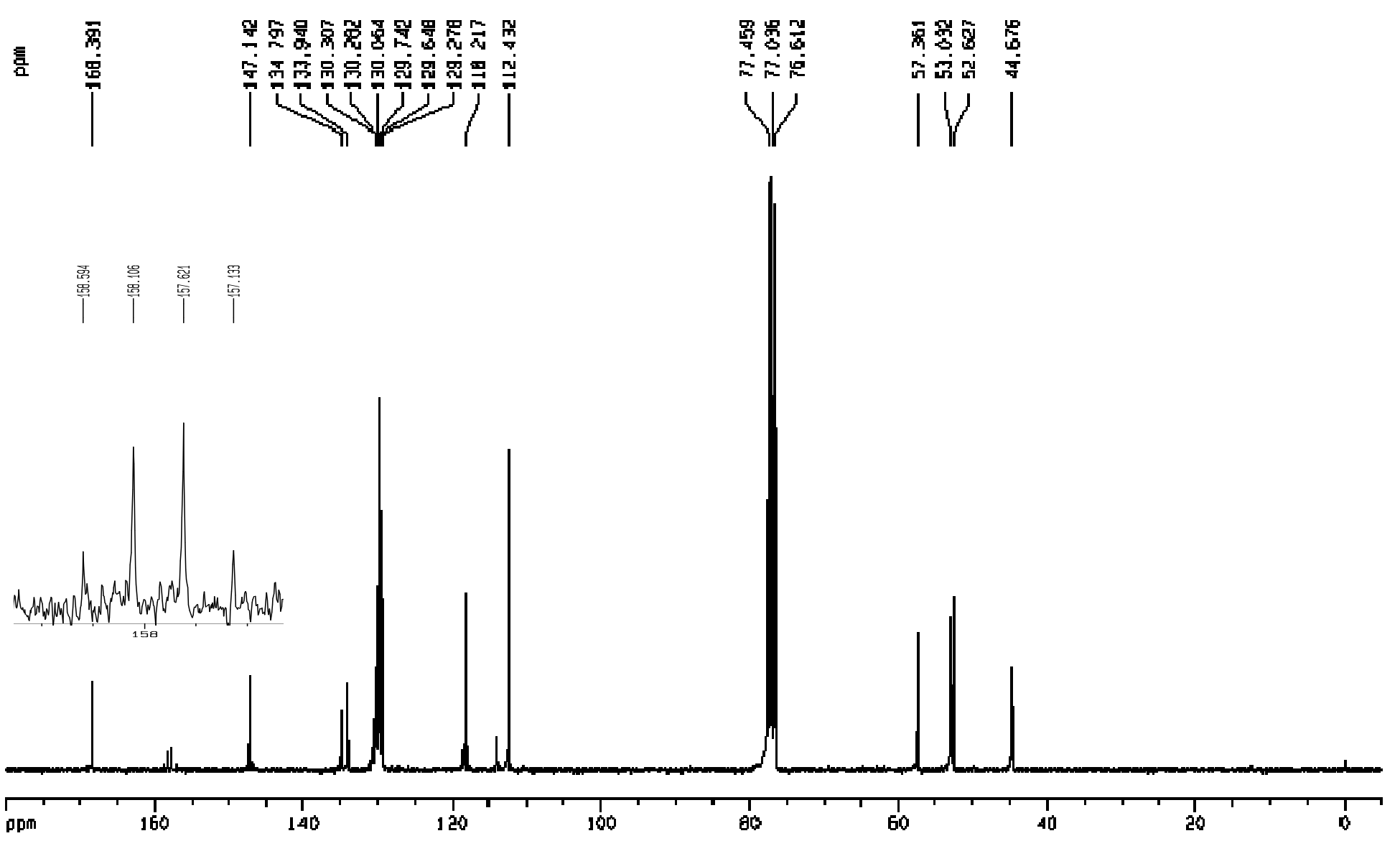

Ourrent Gara Par arecers

MHAE

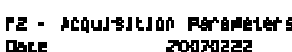

DOLE-

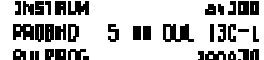

${ }_{10}^{\text {PULPMDO }}$

70
SOLY
TE

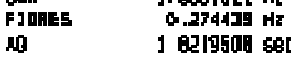

PG 23 日an

TE

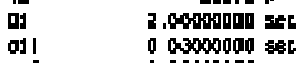

ocotomo set

Mats OHAhtel if -.......

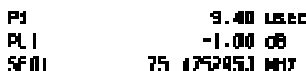

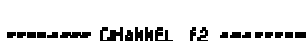

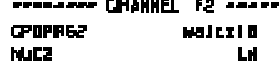

MuL2
PCPO2 LN

$\begin{array}{ll}\text { D.2 } & -1.00 \text {.8 } \\ \text { P.12 } & \text { Ja } 100 \text { of }\end{array}$

Al19 Jat

F2 - Pracessing poraneters

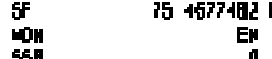

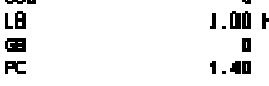

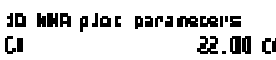

cr da.tio ch

FJP
FJ
Fas

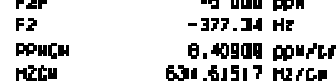


15b- ${ }^{1} \mathrm{H}$ NMR

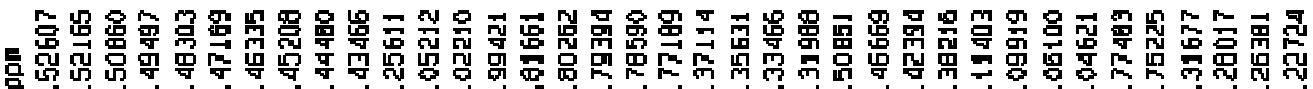

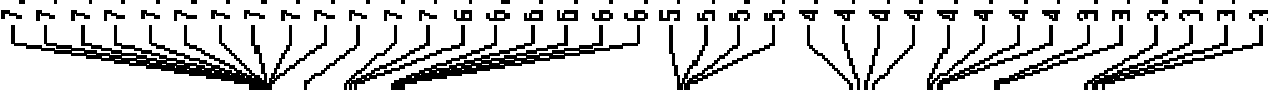

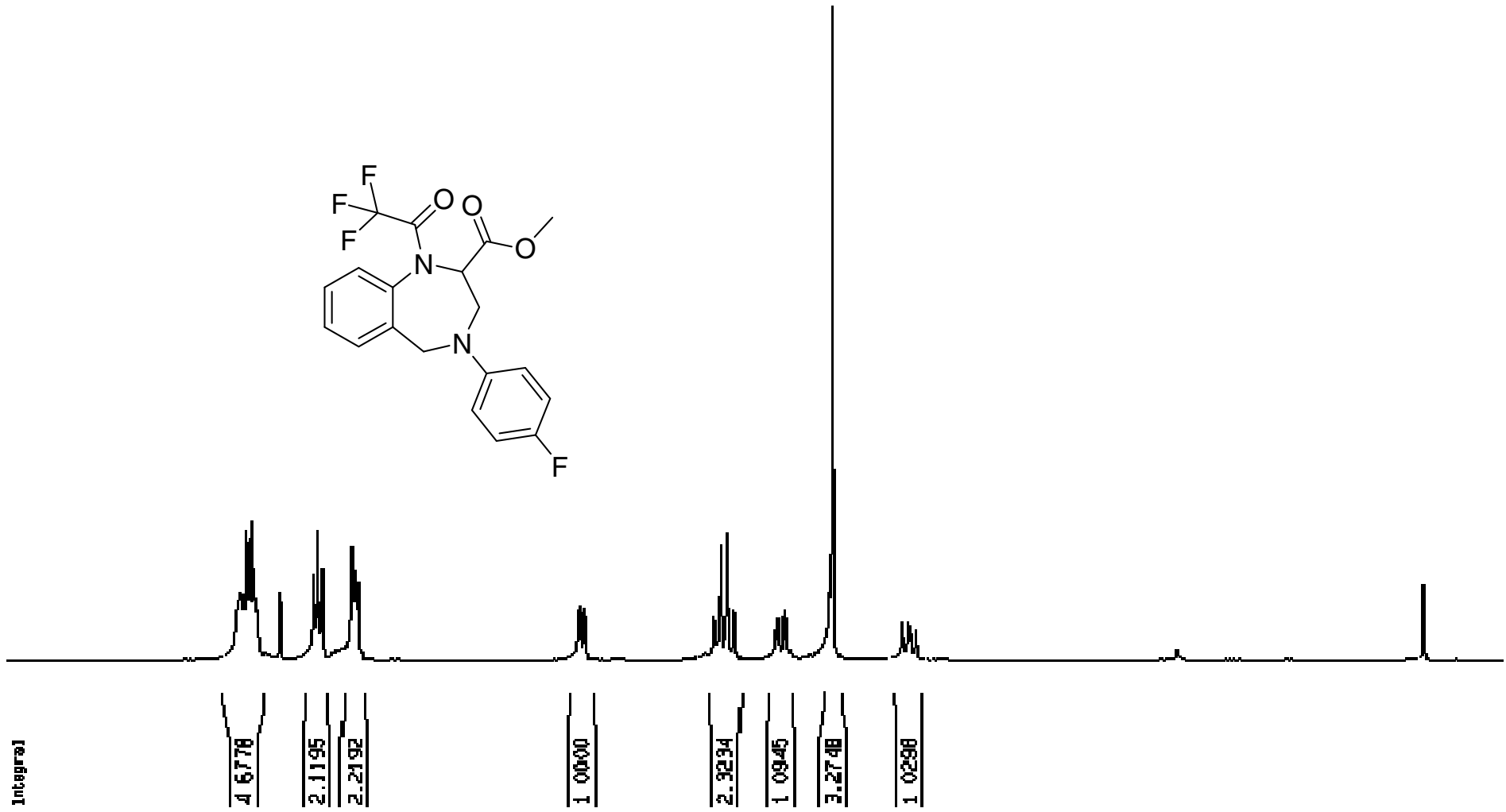

Durrent Date Paraneters

Male MJ Y0T-02] do

PFóch

Jo

FZ - hrgujsjtunn Porameters

Dote-

200 roze10

Intring

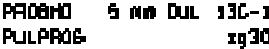

TOL

W5

I5

5HH $6172.899 \mathrm{~Hz}$

ug $5.094190 \mathrm{HI}$

PG 5.306450

a1. Dón usec

5.40 bose

LE
TE
DI
DI

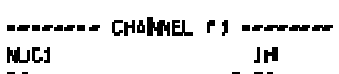

$\begin{array}{lr}\text { Pl } & \text { 9. 30 usec } \\ \text { PL1 } & -1.00 \text { t9 }\end{array}$

Procose ing parangters

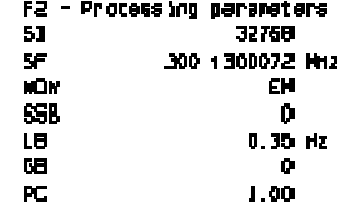

Ló litaf plat paraneters

cox 2a. $00 \mathrm{~cm}$

$\begin{array}{ll}\text { CY } & 90.00 \mathrm{cn} \\ \text { FIP } & \mathrm{g.000} \mathrm{pon}\end{array}$

$\begin{array}{ll}\mathrm{FI} & 2700.17 \mathrm{~Hz} \\ \mathrm{FaP} & -0.500 \mathrm{pan}\end{array}$

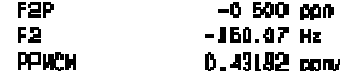

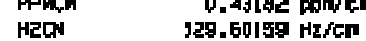


$15 \mathrm{~b}-{ }^{13} \mathrm{C}$ NMR

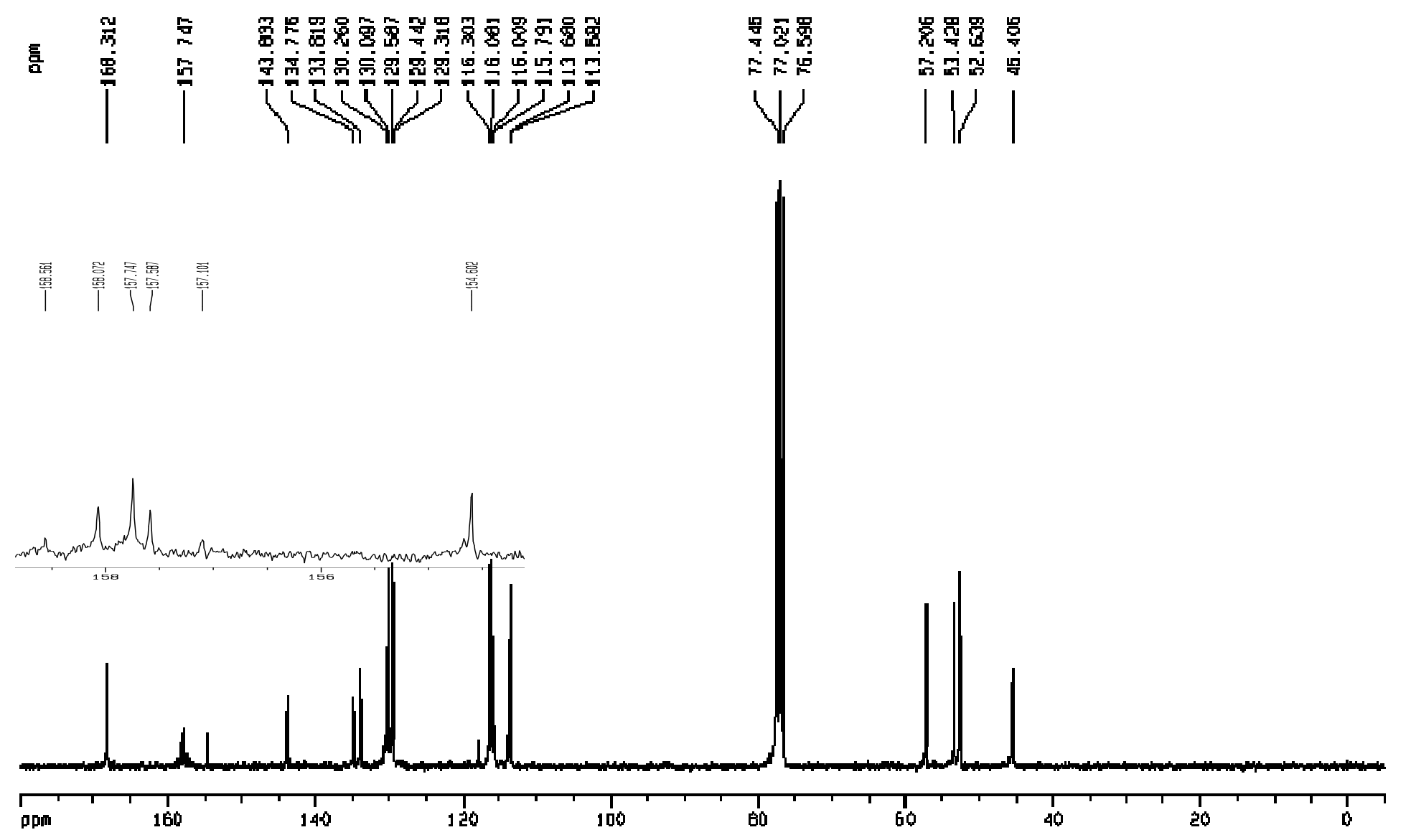

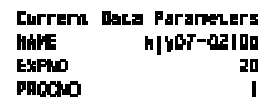

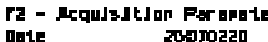

Tute-

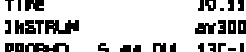

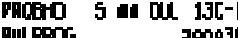

Gollew

I47s

5nH 1 Mog, 611 He

Ho 1 Bejgant $69 \mathrm{C}$

of er.ung user

TE 2 ah $7 \mathrm{k}$

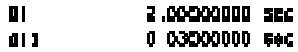

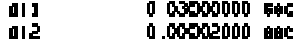

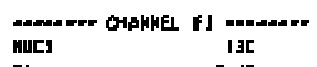

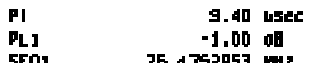

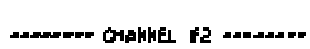

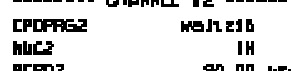

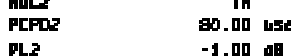

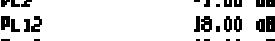

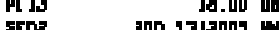

F2 - Frocessinn poranecters

51 - Focessing poranters

Mok" 75 1677

LI 1.0

I0 Wh p por pardnerer 5

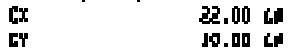

Fiso.

$\begin{array}{ll}\mathrm{FI} & 1394.20 \mathrm{~Hz} \\ \mathrm{FFF} & -5.000 \\ \mathrm{F2} & -377.34 \mathrm{pm}\end{array}$

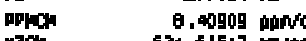


$15 \mathrm{c}-{ }^{1} \mathrm{H}$ NMR

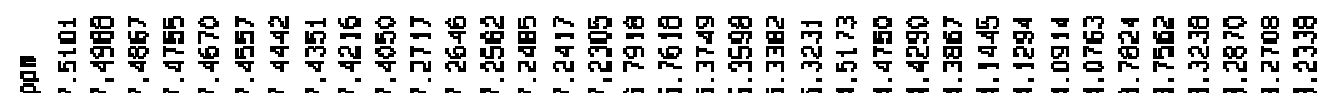
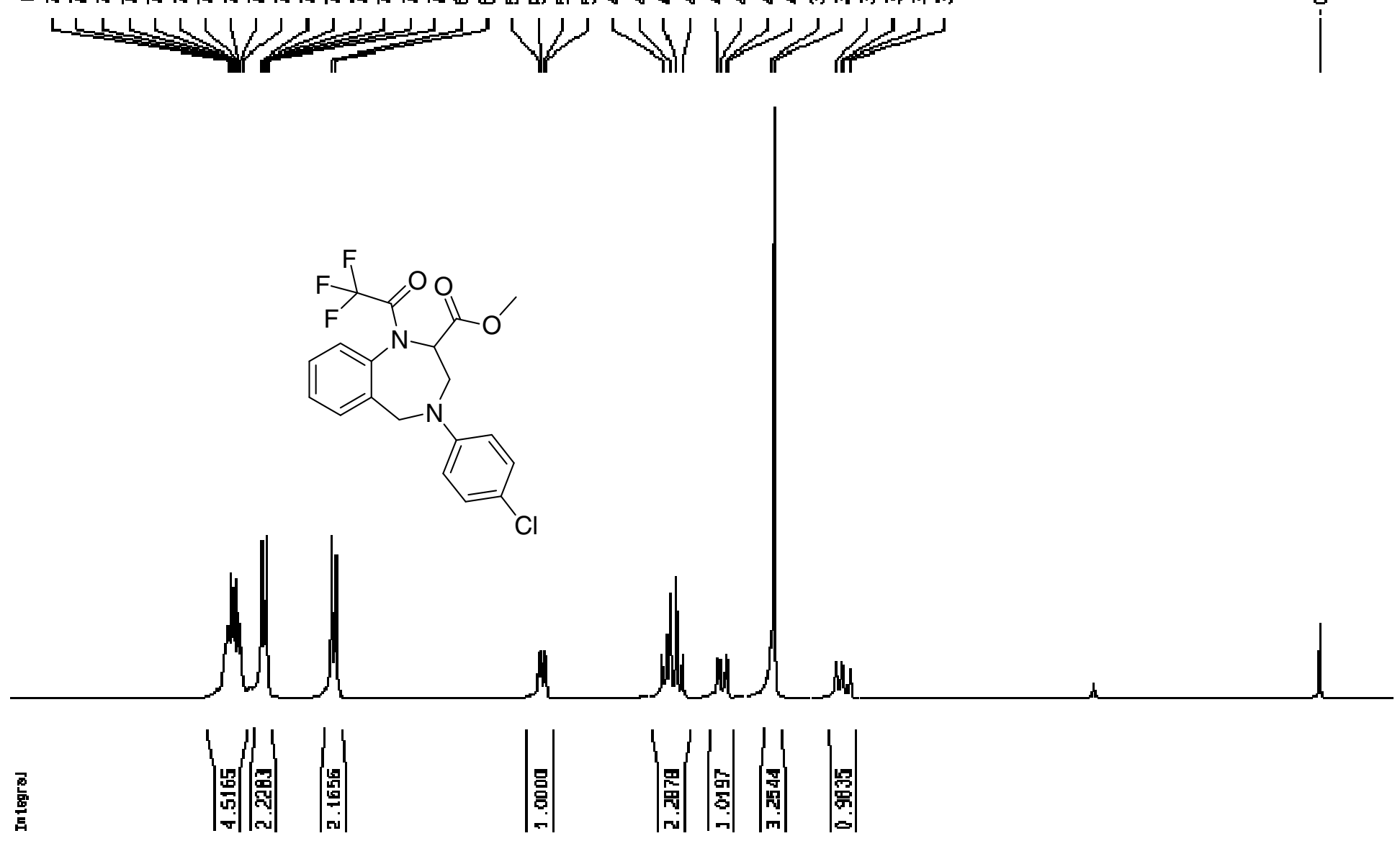

\section{Current Dace Paraneters}

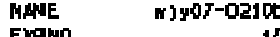

Ppocho

Fe - hraussit jon Parangter

Dace- 200702

7 ine

12.37

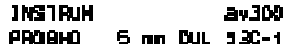

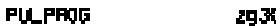

TD

SOL VENT COCI

of $\quad 2160$

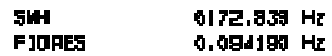

hig 5.3084660 ser

PG 287.4

OE

012.500000000

MLES

PILI

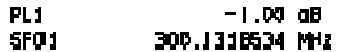

F2 - Pracessing paraneters

51 $350.13000 \% 1 \mathrm{miz}$

INDh

559
녀

J0 Mif p Jot paranetert

C) $22.0 \mathrm{con}$

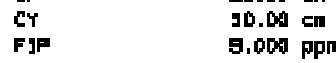

F1 2701.17 Hr

FP

PAMCH D.A3yag ppn/c n 
$15 \mathrm{c}-{ }^{13} \mathrm{C}$ NMR

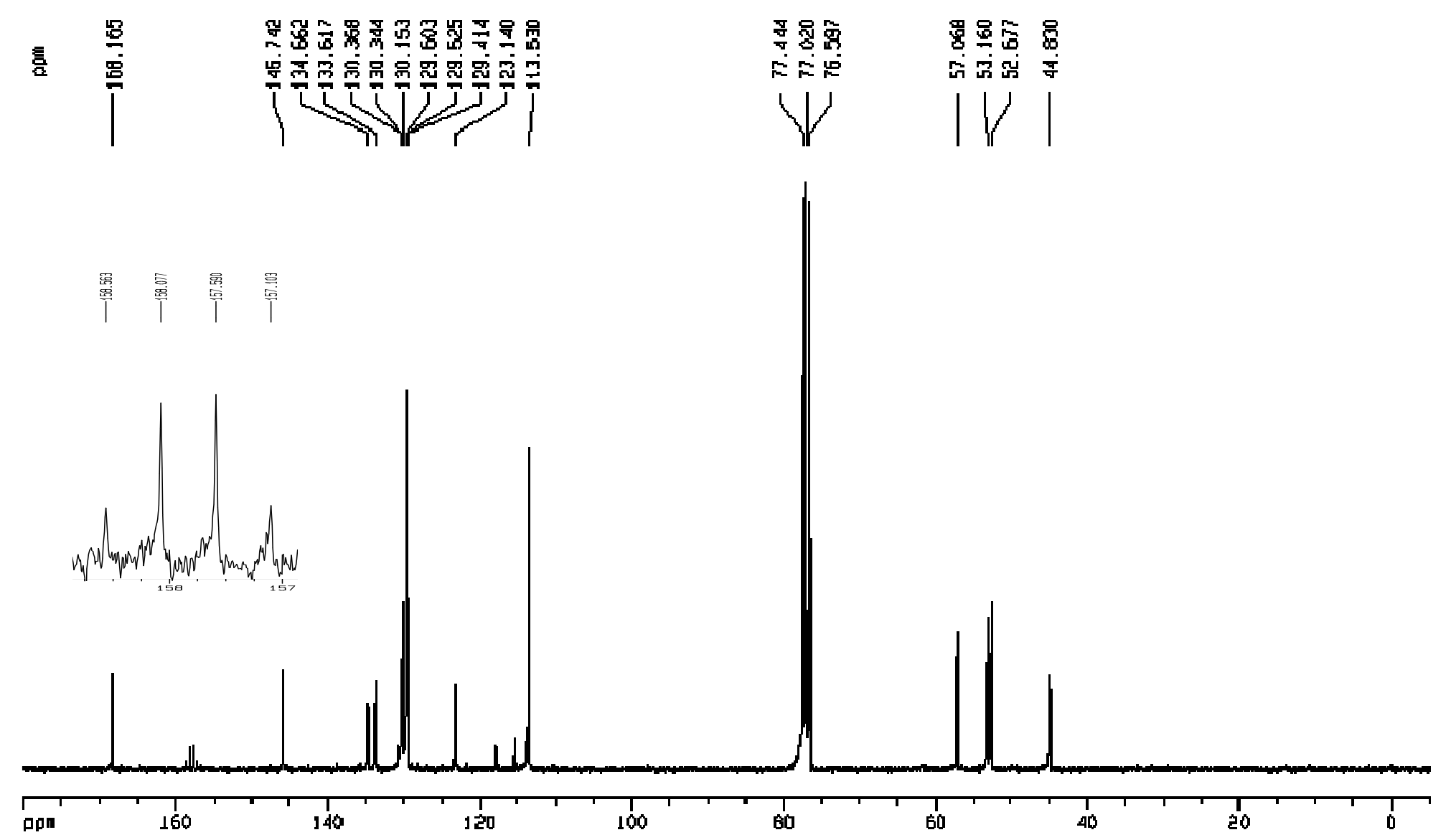

Current data Paranters

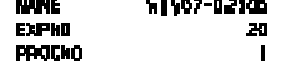

PE - Mequidudun Parengters

Da CE-

IJME

MSint

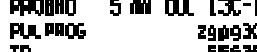

To vew1

56 5033

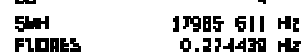

10 182105096

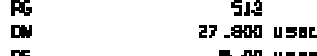

TE

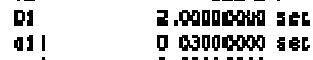

ale

Mag

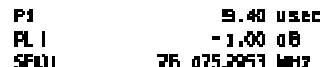

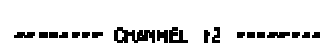

TFDPFG

PrPo?

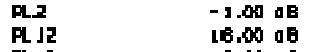

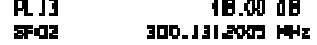

F2 - Proctesung puranecters
51
if

LES

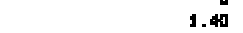

J0 HHF alou geranghers

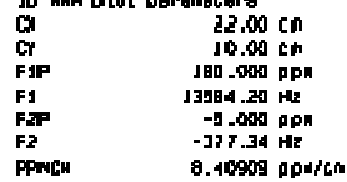

PFwCH $\quad$ a. $40900 \mathrm{pou} / \mathrm{hm}$ 
$15 \mathrm{~d}^{1} \mathrm{H}$ NMR

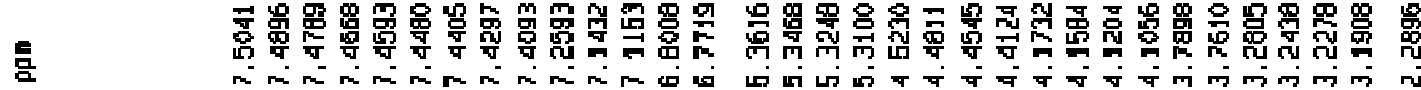

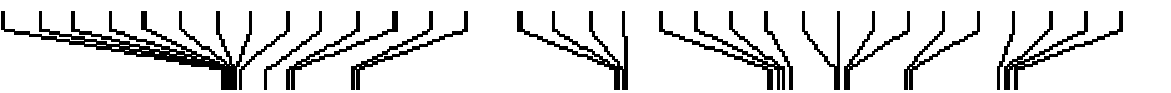

i

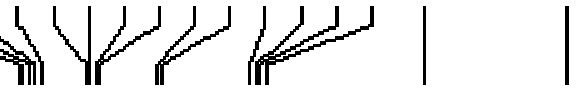

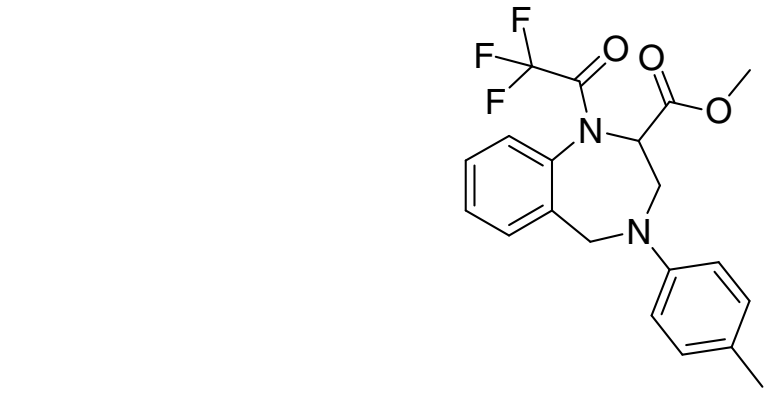

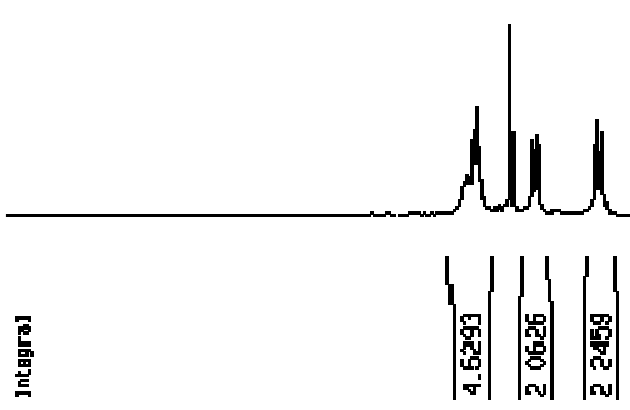

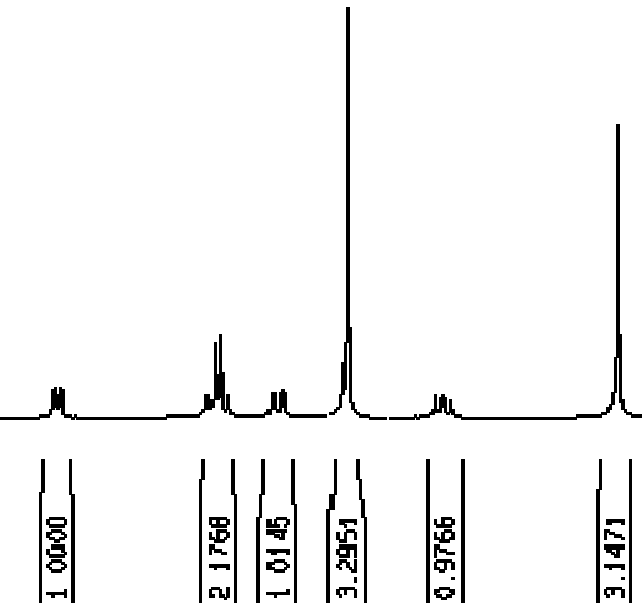

Durrent Deta poryngers

Male gidat-12] gef

PAOCho

F2 - Ncoubsittuan Par oneters

Date_ 2005]2l

Tims

PACOHD 5 on OUL

PMLPFIOS E nT OLL LIL-

To

So

DS

F]DAES D, 1994199 HE

PG 53084660 :

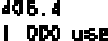

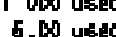
2. 000000005

MLC] CHAMEL II -...-...

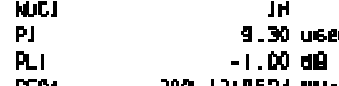

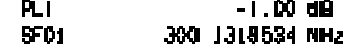

F2 - Processing poranechrs

S1 320 TES

mow

LA

FE

EN

ง.

$1 . \infty$

10 kirk plot paraneters

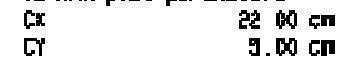

FIP $10.000 \mathrm{ppm}$

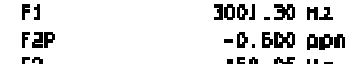

PFUEH

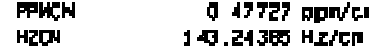


$15 \mathrm{~d}-{ }^{13} \mathrm{C}$ NMR

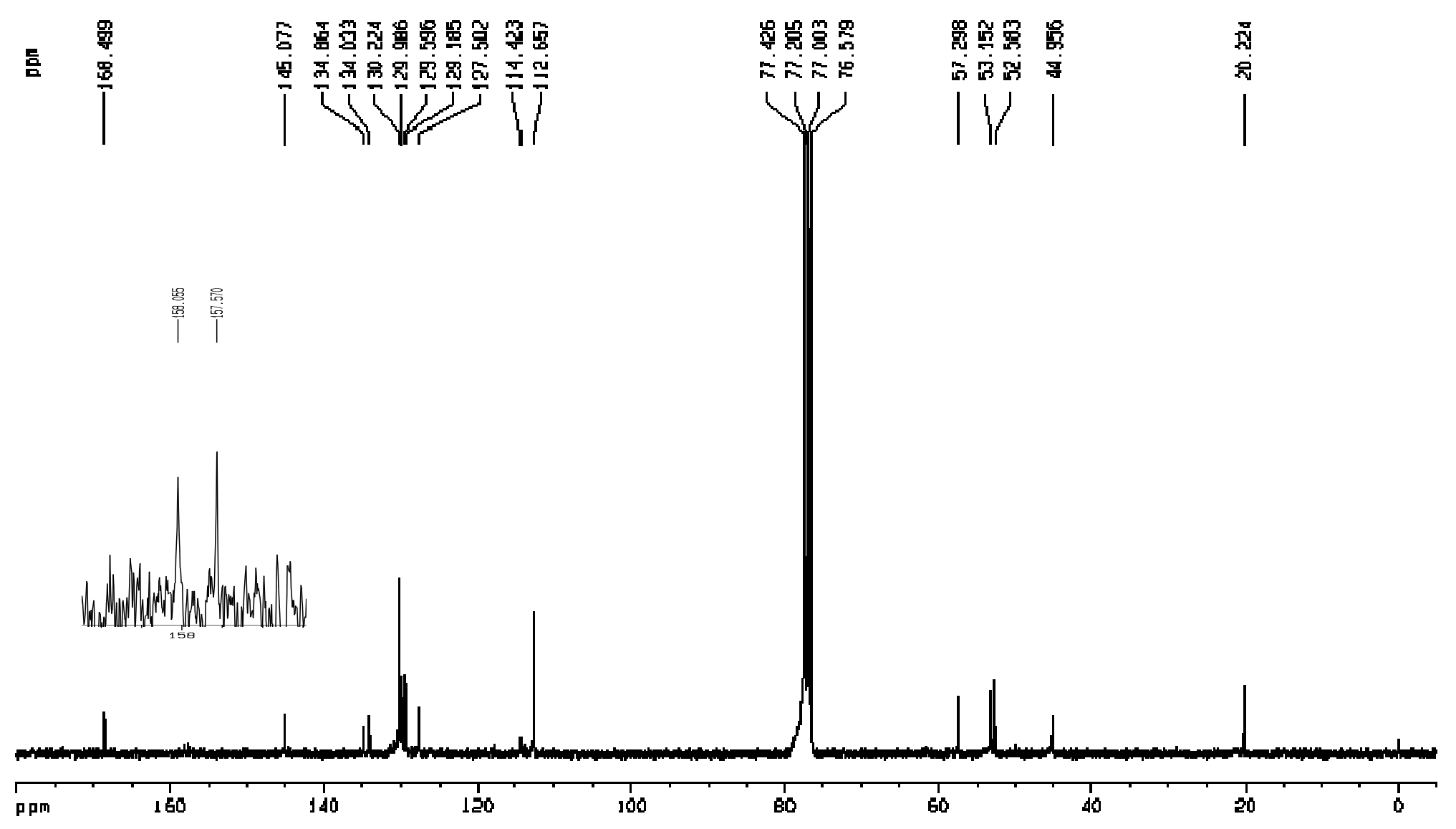

Currenk dota Parometers

EApho

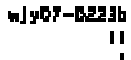

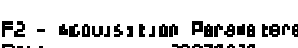

Dath-

[METRN

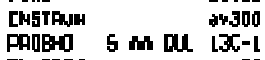

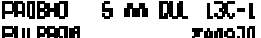

Ta

.6.

J7985-61 ${ }^{1}$ HI

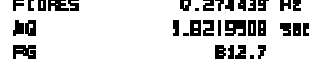

DE 27 \&ant usec

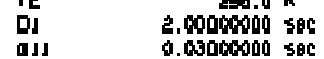

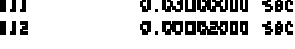

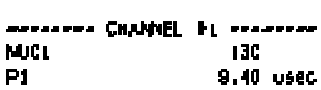

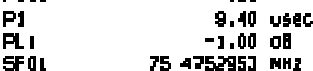

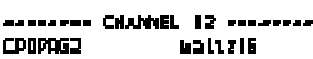

cures bolitif

IE
and an

rz - Pracesa ing peranaters

gir Pracest ing perinnters

S에

LQ

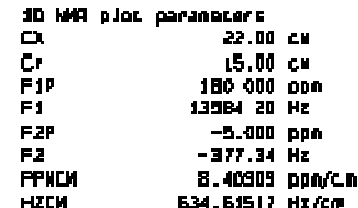


$15 \mathrm{e}-{ }^{1} \mathrm{H}$ NMR

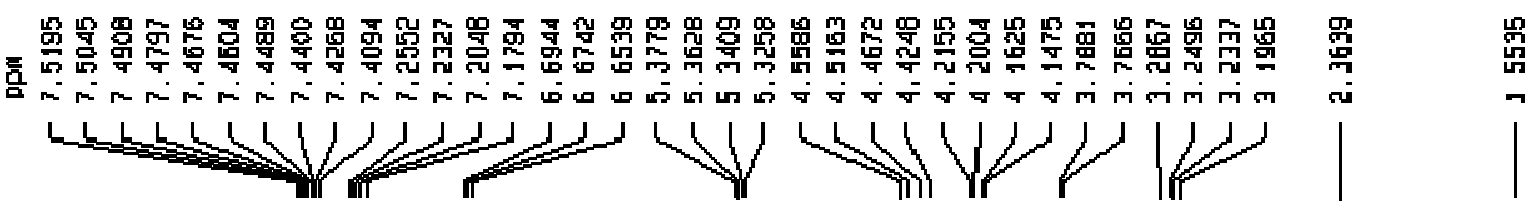

Aurrentit Dothe Poranceters WAME Mystr-0227da (a) 10

Fa - NCquelk ton Paranteters

DOt:

INAS 20.11

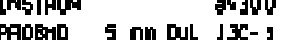

PULPODS

TO $\quad 653.36$

SOL VENT COCIO
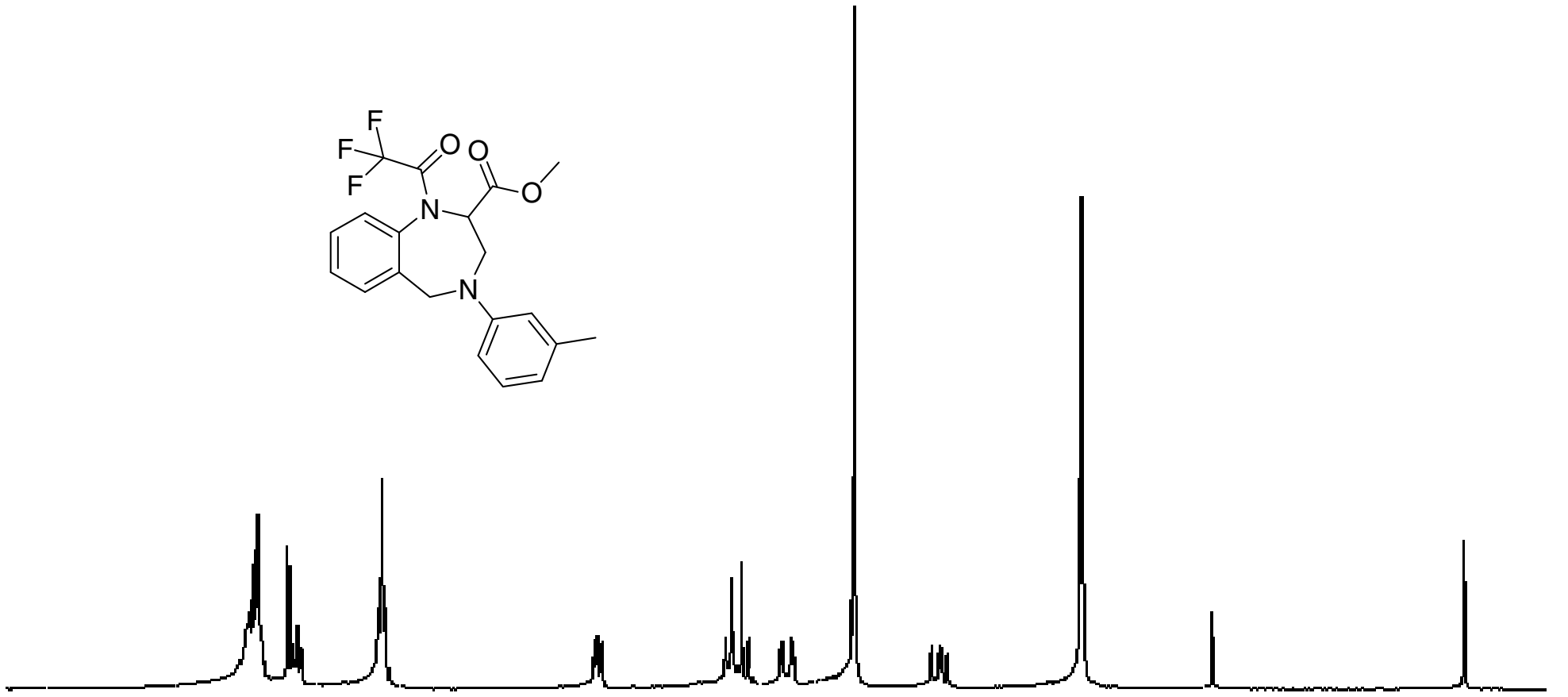

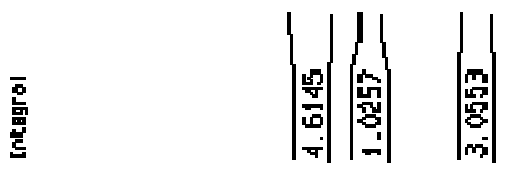

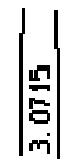

6172 $939 \mathrm{H}_{2}$

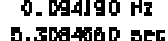

387.4

20.4

6.00 use

6.06 년

2.40000000 set

==-=:== CHANWEL H] ==-==-=-

Hat

P. $\quad 9.30$ usect

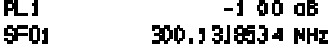

F2 - Procesesng paraneters

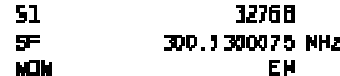

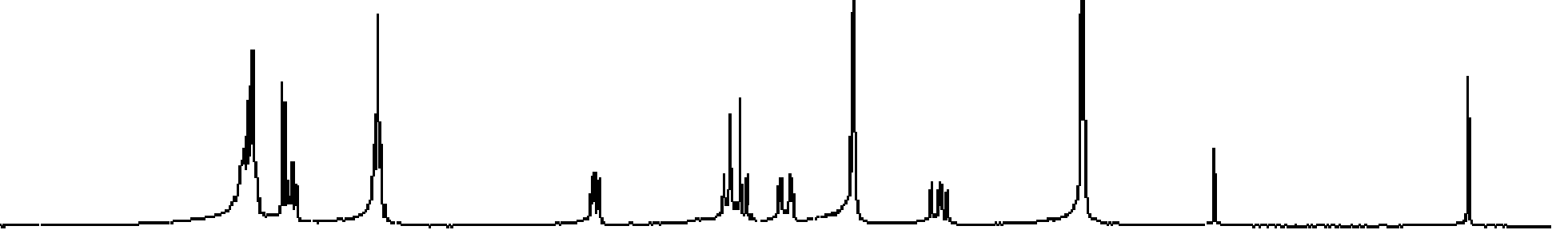

558

PC

EH

IO wh plot paronaters 22.00

$\begin{array}{ll}5 \mathrm{Ch} & 22.00 \mathrm{~cm} \\ \mathrm{Cr} & 10.00 \mathrm{~cm}\end{array}$

9.000 pon

FIP $\quad 201.17 \mathrm{~Hz}$

$F 2 \quad-150.07 \mathrm{~Hz}$

PPHCN

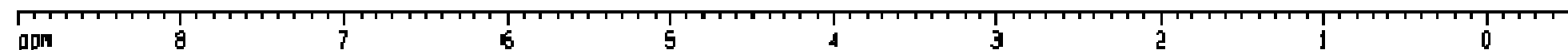


$15 \mathrm{e}-{ }^{13} \mathrm{C}$ NMR

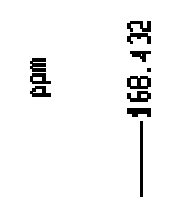

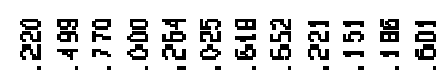

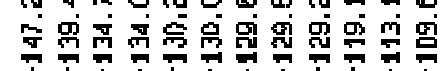

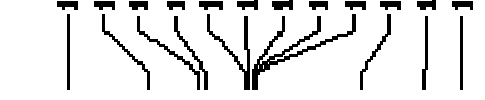

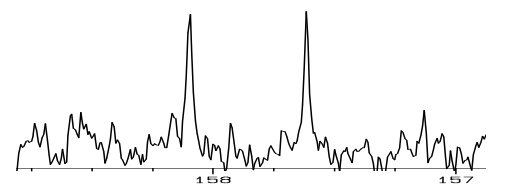

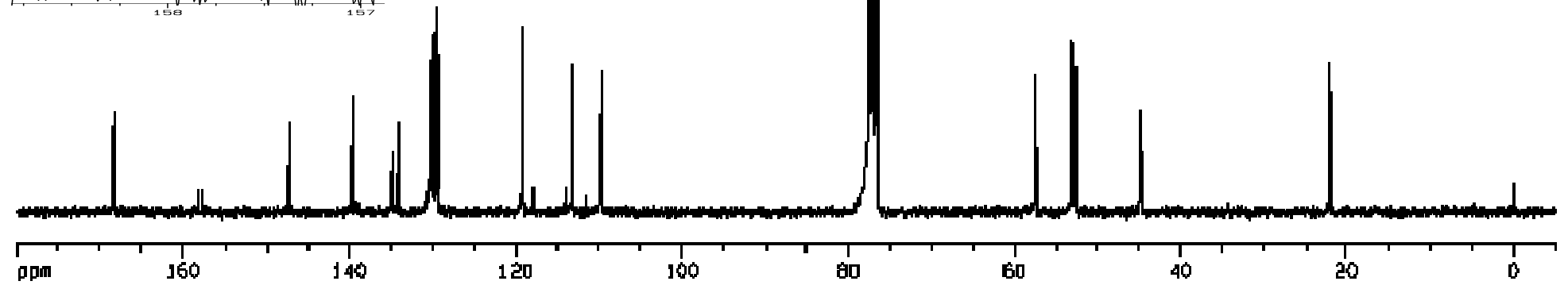

Current Dots Parameters

Expa

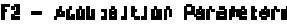

Ture-

JHETAHH $20-17$

PROPOD

pulFor

SOL TEMT
H5

$1 \operatorname{mog} .611 \mathrm{Hr}$

FIDFES B.274-439 $\mathrm{Hz}$

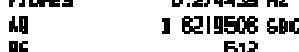

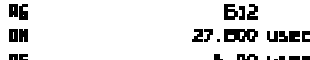

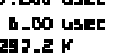

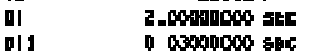

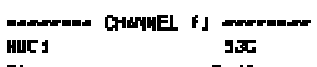

$\begin{array}{ll}P 1 & 9-46 \text { LSEe } \\ P L 1 & -10008\end{array}$

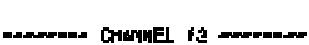

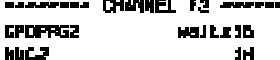

PLFW

$\begin{array}{ll}\text { PLI } & -1-\infty 0 \text { a日 } \\ \text { PLI2 } & 1800 \text { o日 }\end{array}$

$5 \mathrm{Faz} 2000$

Fi - Procesand pararreters

$5 \mathrm{~F} \quad 754677498 \mathrm{HH}$

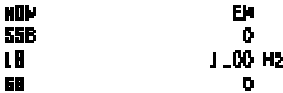

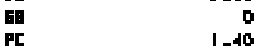

10 Ww pou paramecors

cy
ci

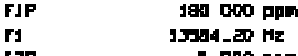

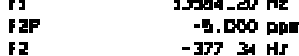

pplach $\quad 0.40909$ pponce 
$15 f-{ }^{1} \mathrm{H}$ NMR

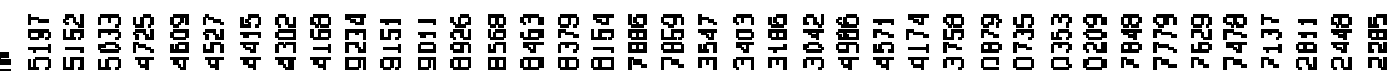

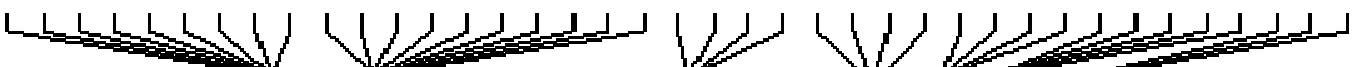

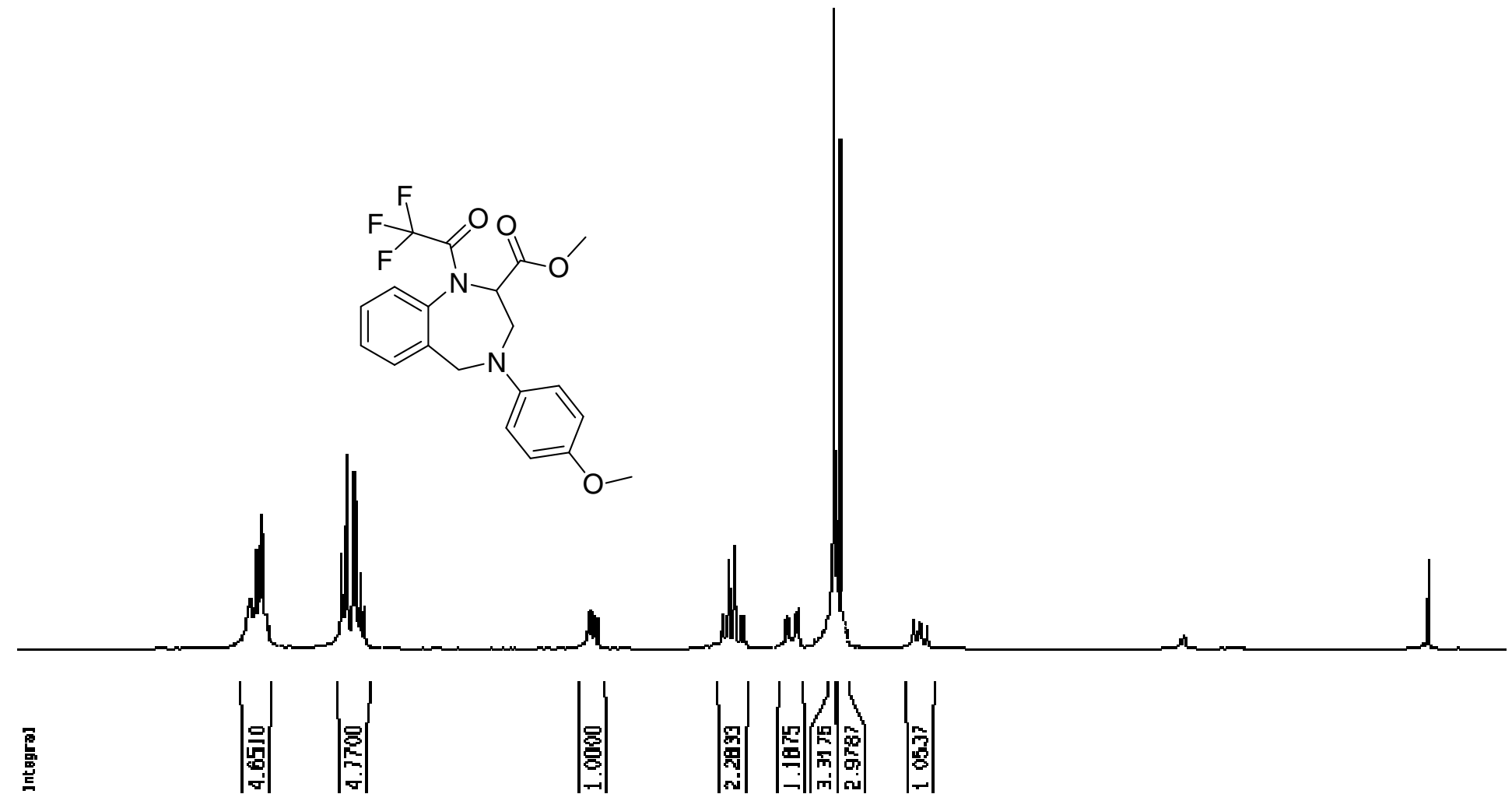

\section{Gurrent Daca Paranstere}

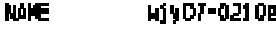

PAocko

I

F2 - Acnuls JkJon Poranter

Dete- 201702

Int

IMSTFLW

PAcent 5 nn DuL ISE-1

PULPAOG

SOLVEM TOM

$\begin{array}{ll}\mathrm{Og} & 2 \\ 5 \mathrm{H} & \text { 6172. } 939 \mathrm{~Hz}\end{array}$

FIDAES O

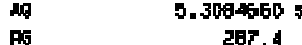

DII Bd 5. GB use

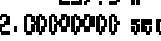

-...-..- DHAMEL 11 -..-...

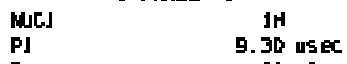
PLL $\quad-1.0048$

F2 - Prncessing parenelers

5] $3276 \mathrm{~B}$

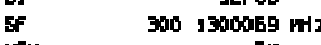

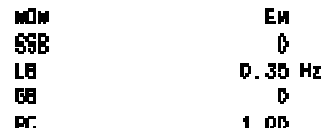

IO MHA p lak par aneters:

Con 22.000 th

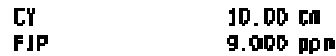

FJ $2001.15 \mathrm{HT}$

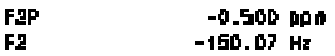

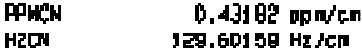


$15 f-{ }^{13} \mathrm{C}$ NMR

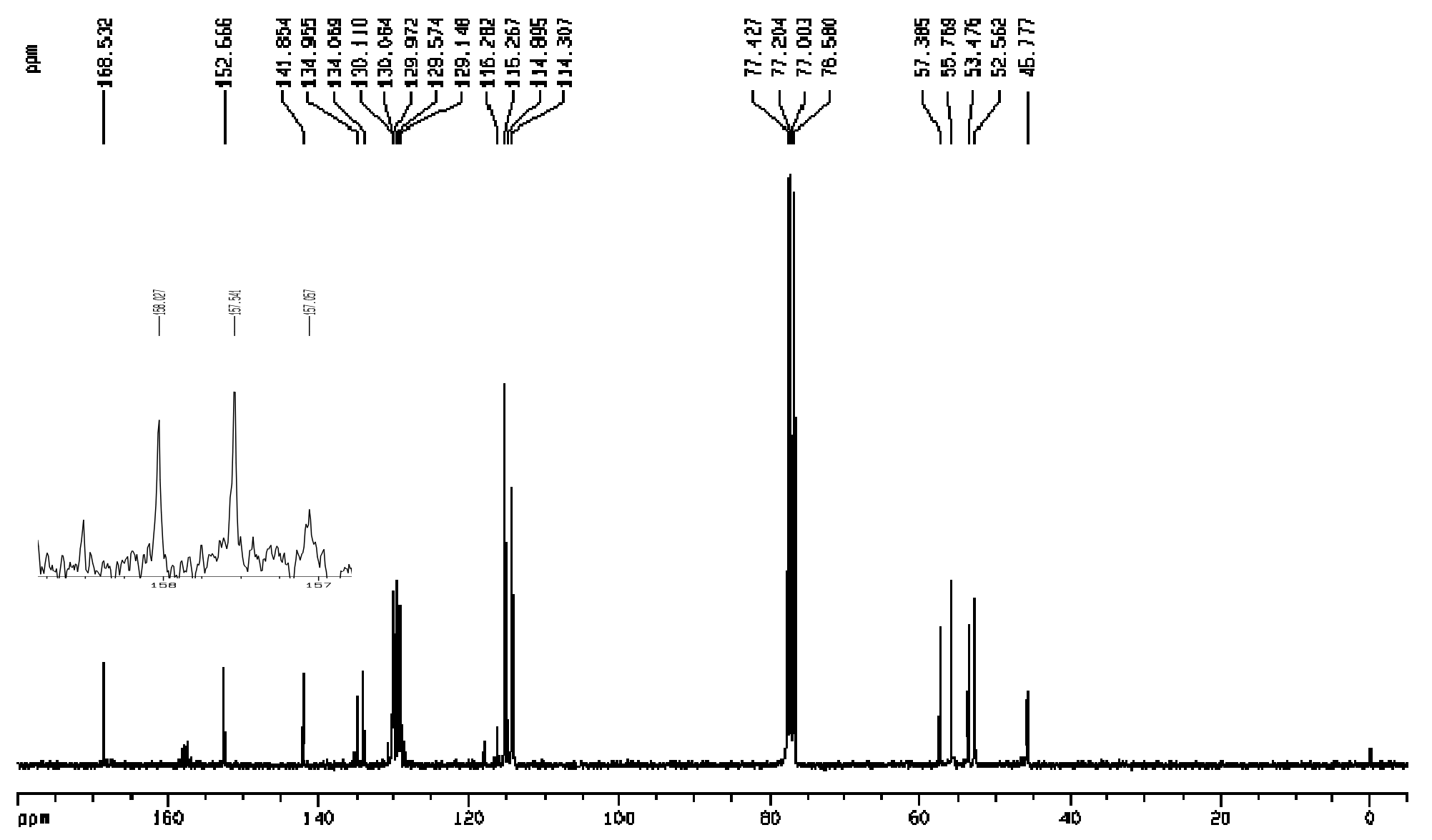

Grrerot Dan Paraneter:

Mink

paroun

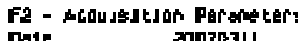

Date-

JMSTRA

PyLFPO

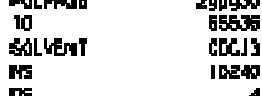

FIITHES 17985.611 $\mathrm{Hz}$

49 $\quad 1.8210509698$

매 27.000 ugec

TE $28.9 .1 \mathrm{n}$

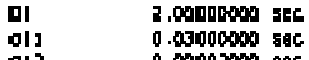

are

MII

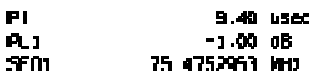

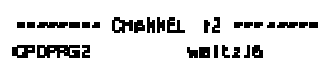

GPOPG

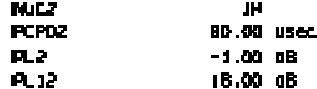

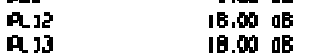

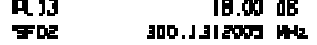

F2 - Processind paranec ers

LOM

la uw p plat parsoncars

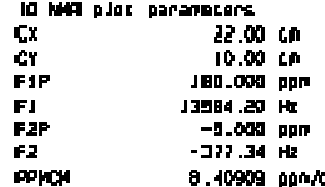

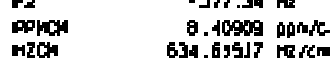


$15 \mathrm{~g}-{ }^{1} \mathrm{H}$ NMR

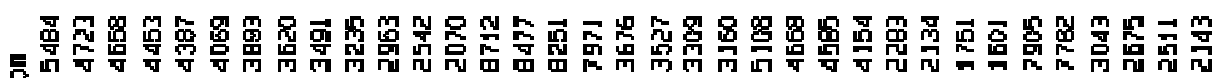

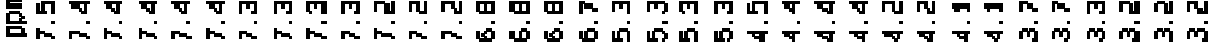

$L=L=L=2$

Drrent Dote Paranec ar:

MUE HIYDT-b3]Ba

PAOCMO

Fi - Menusajtan Parminters

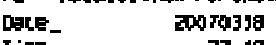

Tine

IM57fut

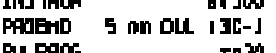

PUPAOC

50.4Ekn mir.13

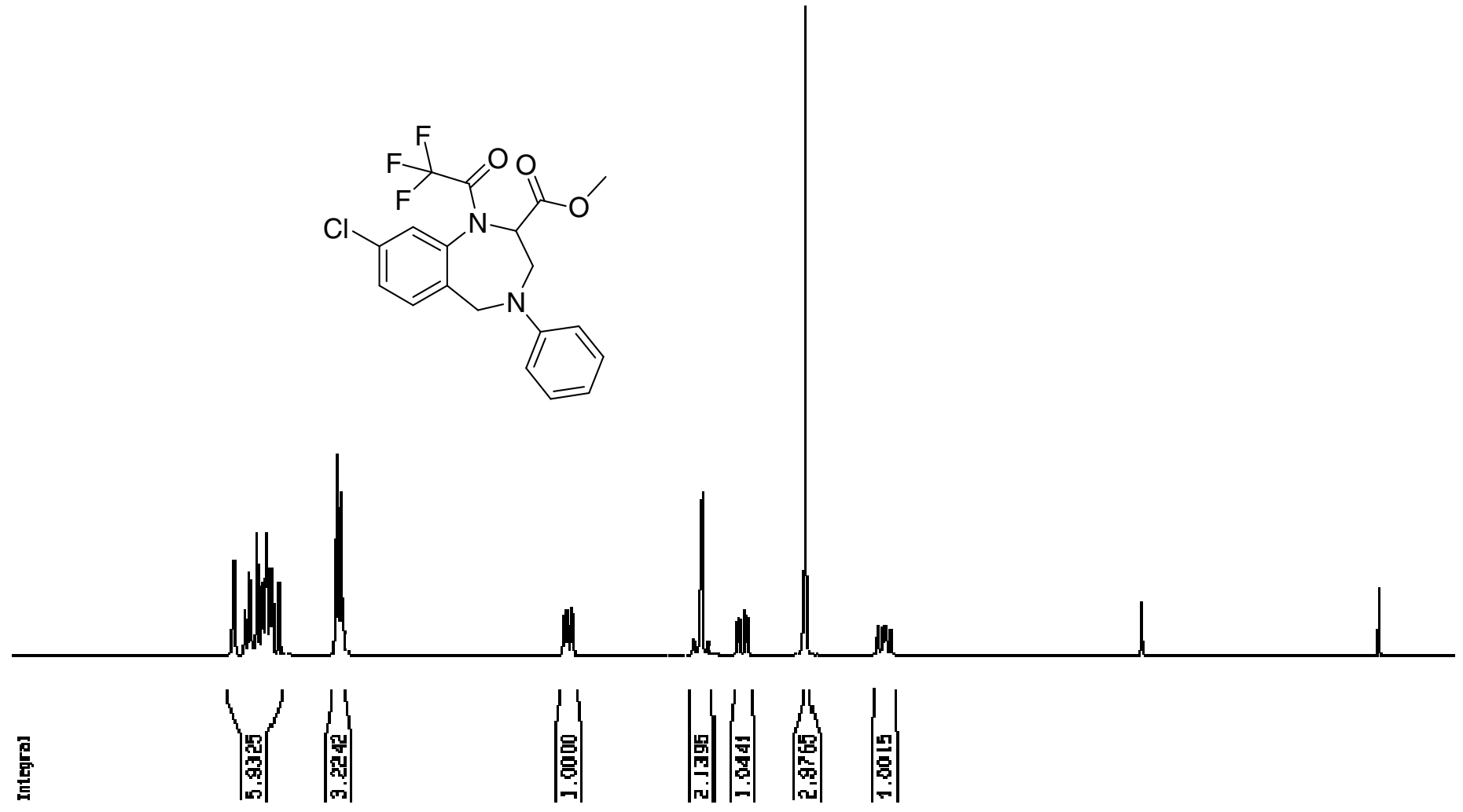

50.4Ekn
D5
D5

BH+1 $0992.006 \mathrm{HT}$

D. I972⿰ Hx

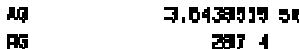

PG $\quad$ Bos t

600 use

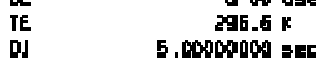

Mag
$\mathrm{P1}$

P1
PLJ

9009

Fe - Prorefsing paranechre

5] 32369

55 30.J3000 $\mathrm{Nm}$

$\begin{array}{ll}\text { SO } & \text { LA } \\ \text { LE } & 0.30 \mathrm{~Hz} \\ \text { PC } & 1.00\end{array}$

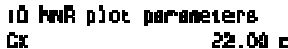

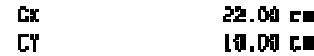

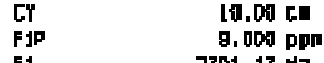

F1 2761.17 Hz

$-6.500 \mathrm{ppm}$
$\mathrm{Fa}$

PAMCN $\quad 0.43109 \mathrm{ppn} / \mathrm{cm}$

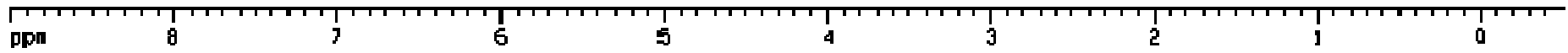


$15 \mathrm{~g}-{ }^{13} \mathrm{C}$ NMR

|
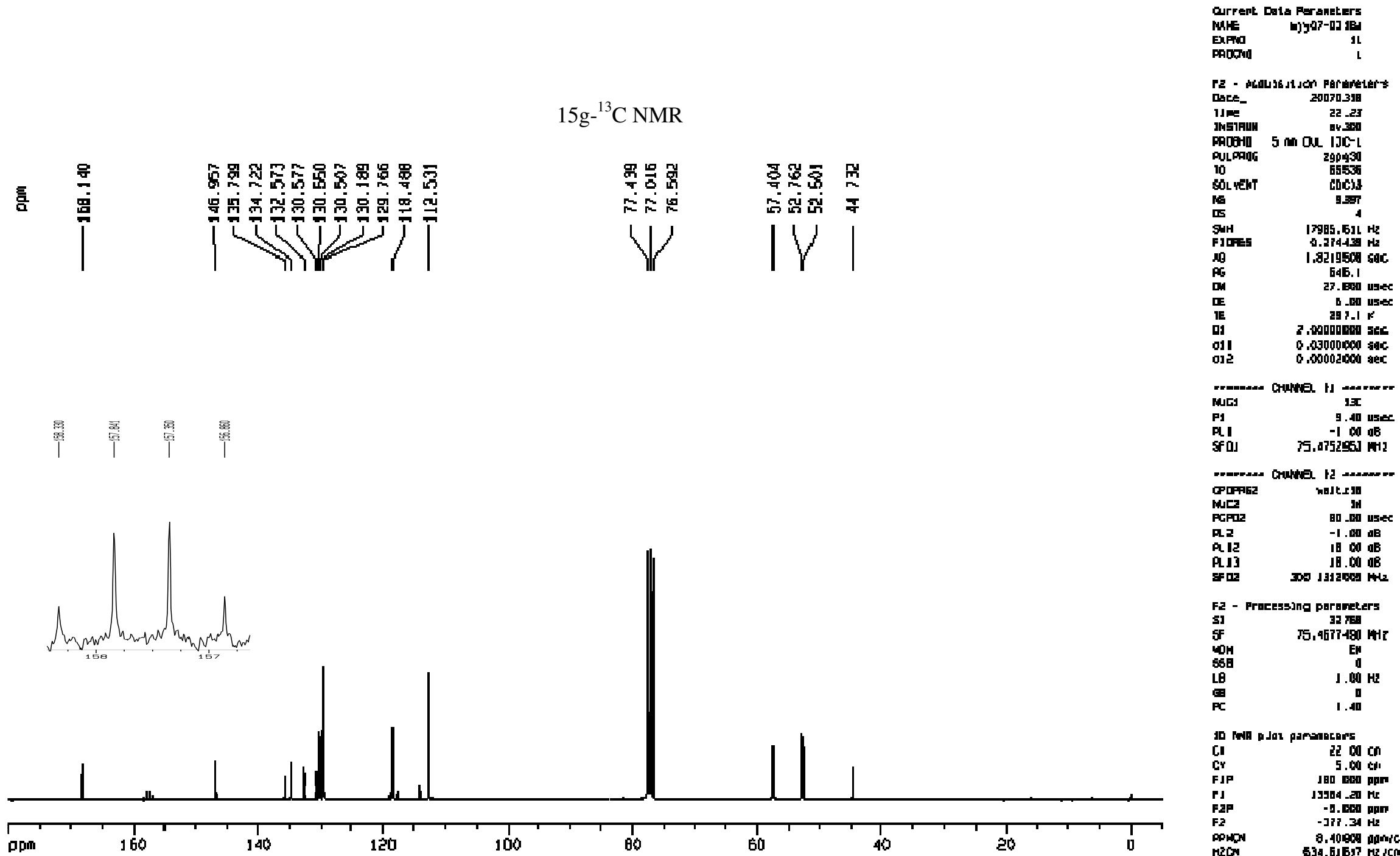
$15 \mathrm{~h}-{ }^{1} \mathrm{H}$ NMR

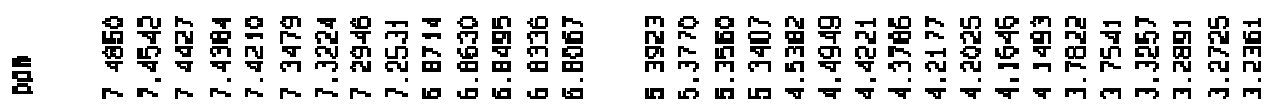
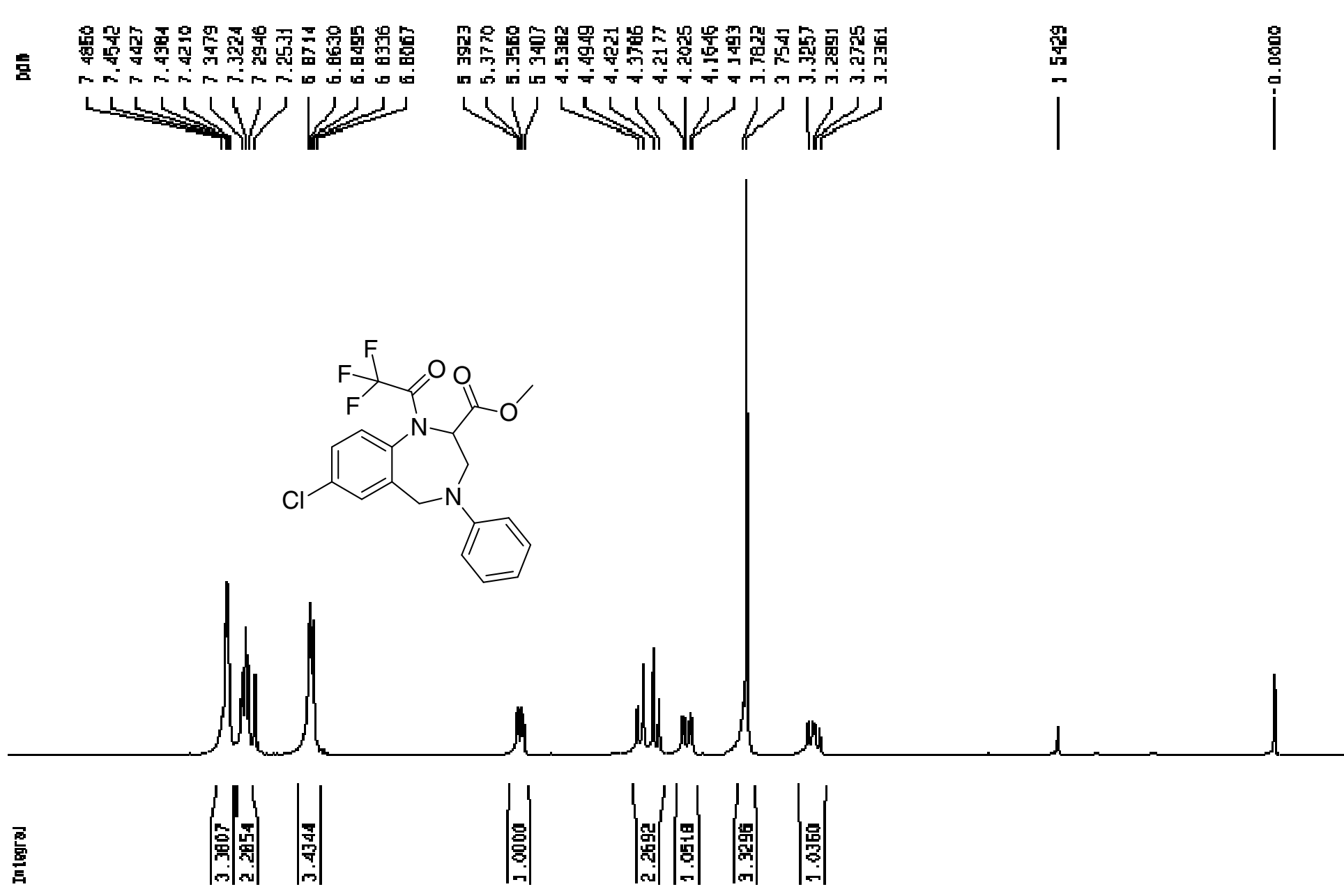

Current Dace Parangters

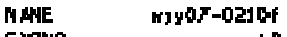

PAOGHO

F2 - dedusajtjon Paranaters

Dace_ 20070210

I me

15.39

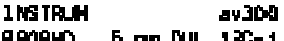

PUPPO5 2930

ID 60035

ins

5it $\quad 5172.835 \mathrm{~Hz}$

AG $0.09 \mathrm{~g} 19 \mathrm{NO} \mathrm{HI}$

$\mathrm{AQ} \quad 5.30846 \mathrm{BO}$ SE

AG

OE

gi.000 user 6 ON $45 \mathrm{E}$

IE $2.00000001 \mathrm{kec}$

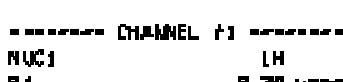

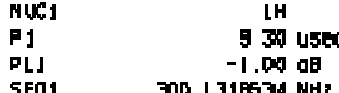

Fa - Pr acessing paruneters

51 32ग0

SF 300.I 30006 NH

IIDK

농

69

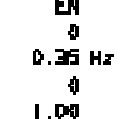

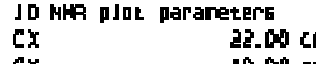

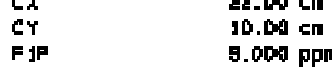

$\begin{array}{lr}F 1 & 2701.17 \mathrm{HI} \\ \text { FI } & -0.5600 \mathrm{gph}\end{array}$

PAUCH

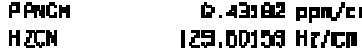

D DIII

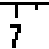

6

$5+1$

3

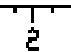


$15 \mathrm{~h}-{ }^{13} \mathrm{C}$ NMR
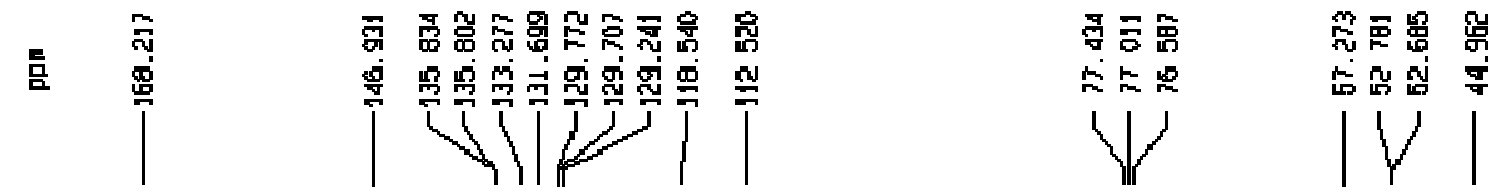

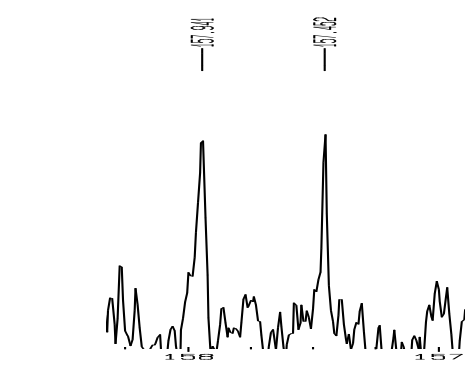
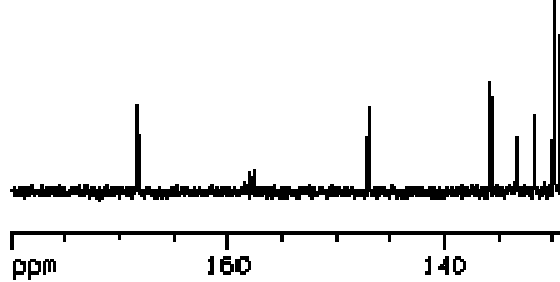

160
140

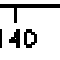

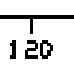

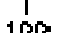

10

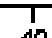

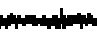

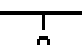

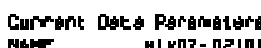

Expont

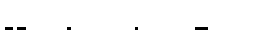

Fa - McquJE Jcion Parseneter

arte-

1540

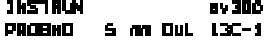

PULP005

STL WEA

55 5 J70

s 1 (t)

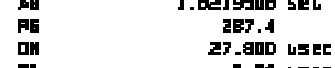

ㅁ ‥DO USE

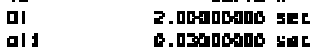

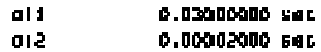

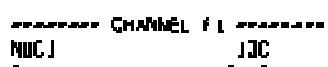

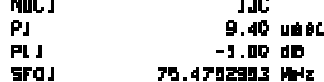

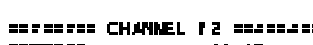

CPOPAG2 MalizIS

PCFOa

PL2

PL13

LE.00 $\mathrm{GE}$

300,1310005

fe - Proceesing darameterte

5123276

50M

28

10 Mur p loc par meerers

$\mathrm{Cx} \quad 23.00 \mathrm{~cm}$

FuP $\quad 1090.000 \mathrm{mot}$

Fap $\quad-5000 \mathrm{hot}$

${ }_{\mathrm{HZOH}}^{\mathrm{PPHCH}} \mathrm{E}$ 
15i- ${ }^{1} \mathrm{H}$ NMR

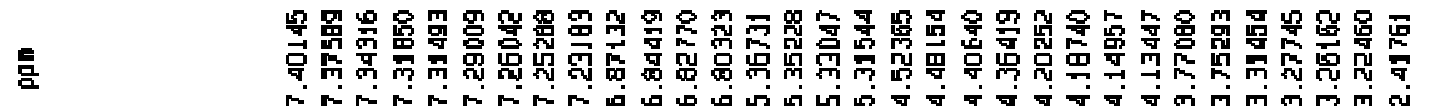

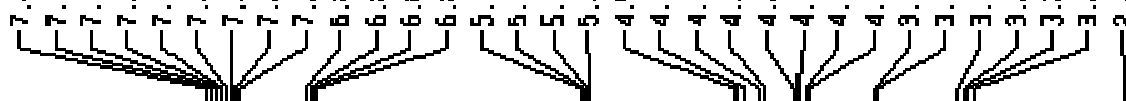

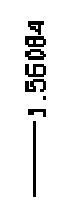

Durrent Dek poranecers

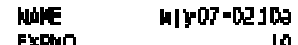

PaOcho

F2 - Acaubsit I Ion Par aneters

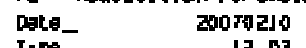

Tum

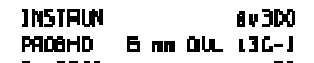

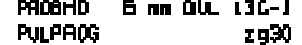

Pyl

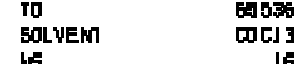

W5

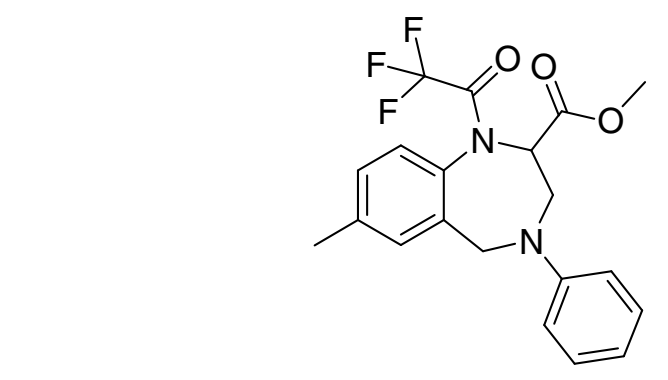

5NH

ig

PG 286

IE

TE 2. A2G.6 K

MIC] CHOMEL II -...-....

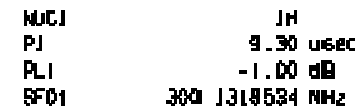

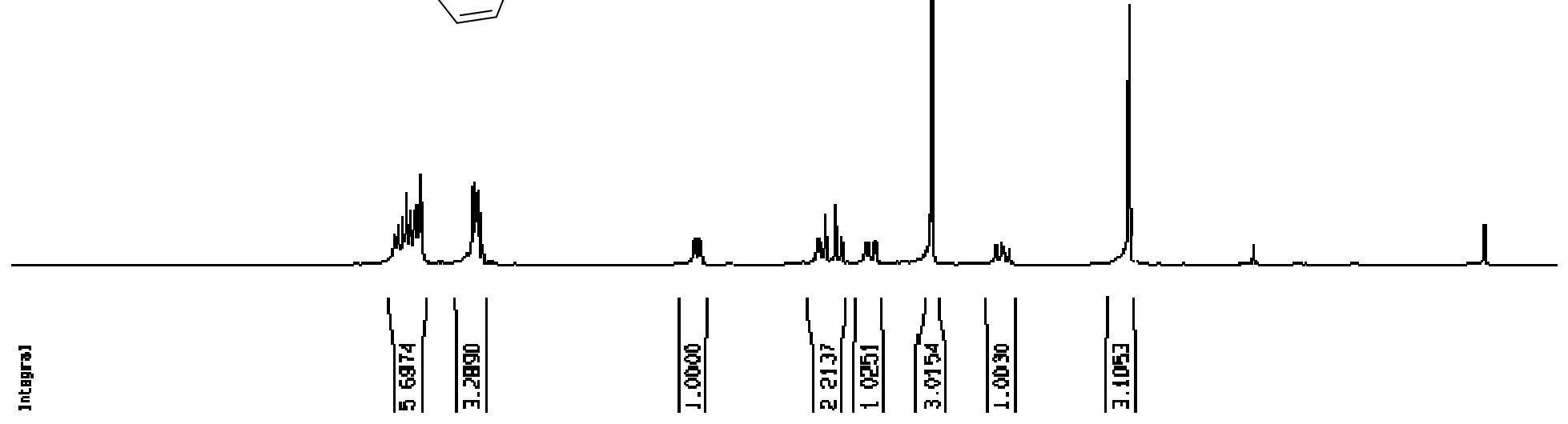

2 - Procensing poranecters

51 T369

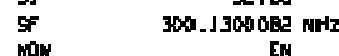

Man

$\begin{array}{ll}\text { LE } & 0.50 \mathrm{HI} \\ \text { PC } & 1.5\end{array}$

10́ kimk plat paraneters

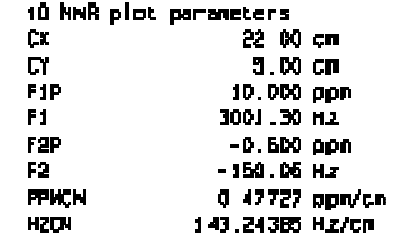

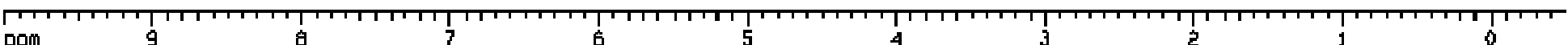




\section{$15 \mathrm{i}^{13} \mathrm{C}$ NMR}

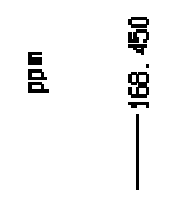

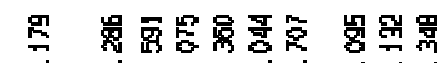

i

\section{pinting}

ppm

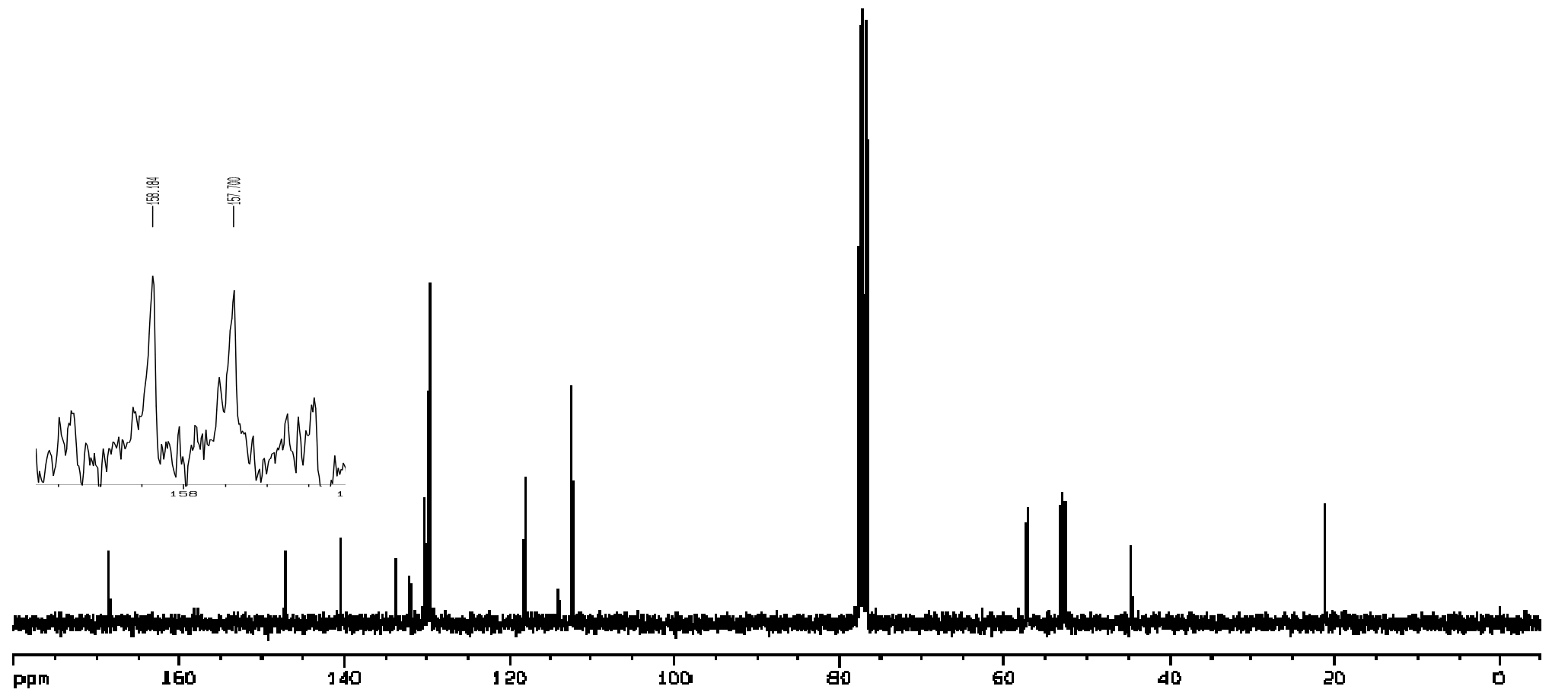

F2 - ACqu IS IL Son Paremgleer a

TIME

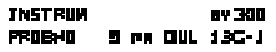

PULFaE $\quad 200030$

TO

SOLY

DS
SWH
FT
Fes

FHH $\quad$ J7905.611 Hz

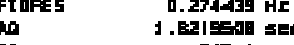

Fit

TC

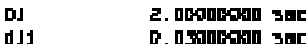

dJa 8.0602000 sac

Wuld

$\begin{array}{ll}\text { P1 } & 9,40 \text { ufes } \\ \text { PLI } & -1,000 \text { of }\end{array}$

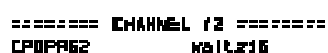

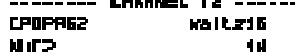

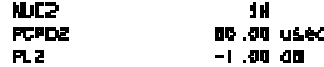

RLIE 18.00 a

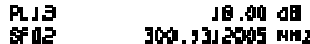

F2 - procosseing par anocere
EI

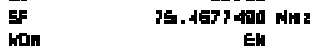

E⿱
LEg

10 Holl a Joe parmenetars

arde 22.00

Fi

F2p

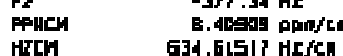


$15 \mathrm{j}-{ }^{1} \mathrm{H} N \mathrm{NMR}$

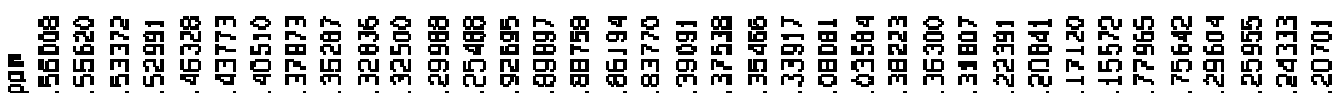
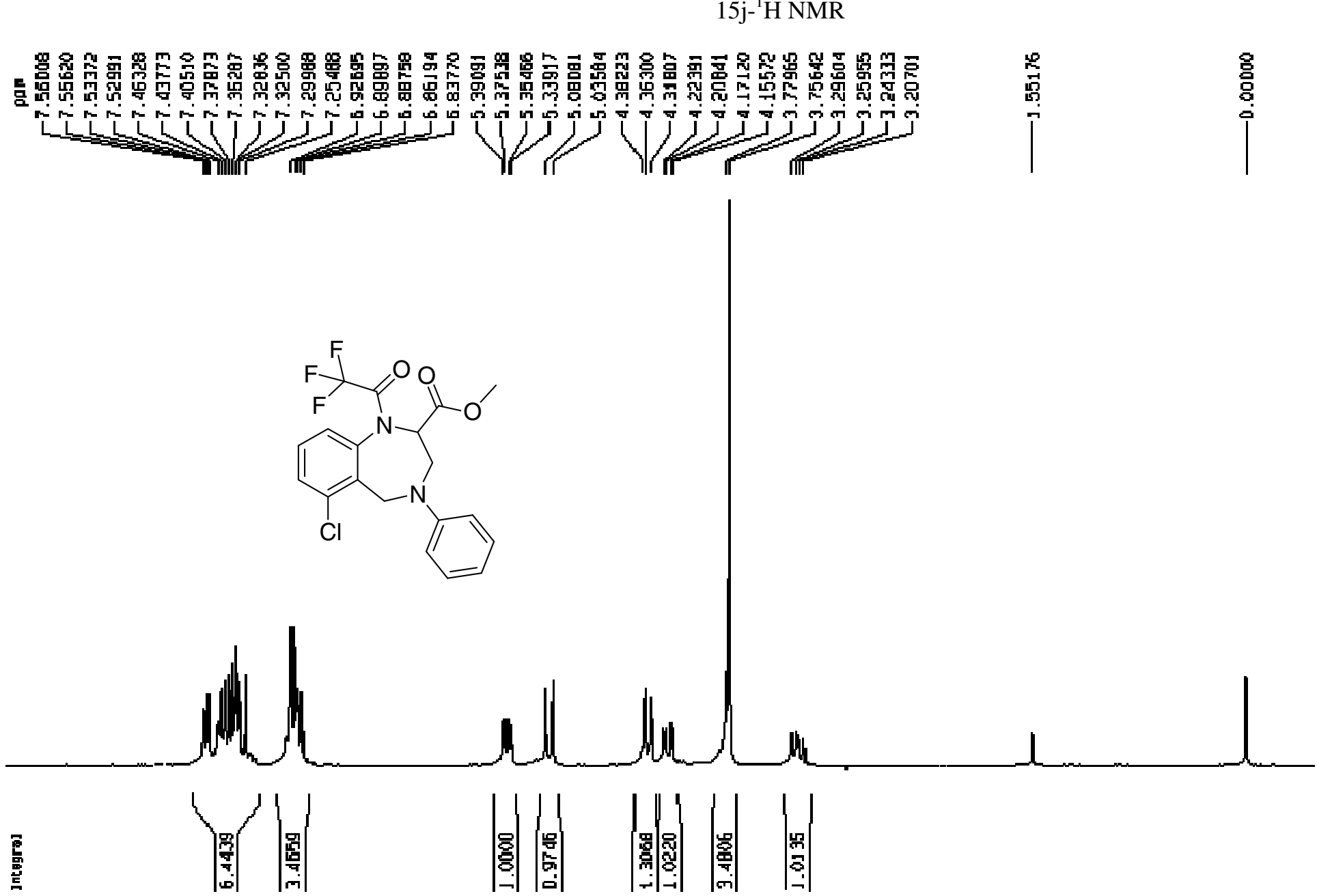

Current: Date Parspigers

HINE

PFOCMO

Fz - kcauss It I on Poronecers

Date- 20070210

7une

PulPAOG

SRL HEMT

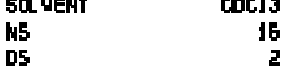

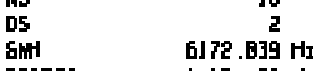

FIOFES $\quad 0.099190 \mathrm{~Hz}$

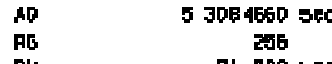

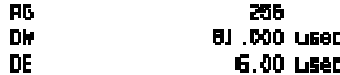

DI z.000000010 Sor.

MUCI CHAMEL II -.2.-.-.

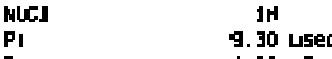

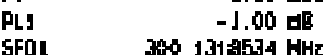

F2 - Processing perangtere

5] 32768

SF WW

559

$\begin{array}{lc}\text { LE } & 0.35 \mathrm{H} \\ \text { IE } & 0 \\ \text { PE } & 1.90\end{array}$

9D kMA plot parsneters

G.

$\begin{array}{ll}\text { Gir } & 10.00 \mathrm{ch} \\ \mathrm{FJP} & 9.600 \mathrm{ppn}\end{array}$

FJ 2 201.17 Hz

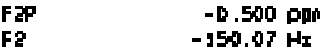

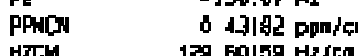

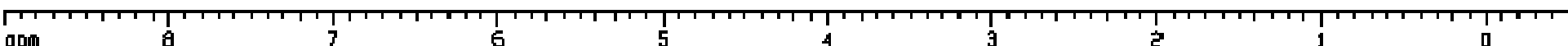


$15 \mathrm{j}-{ }^{13} \mathrm{C}$ NMR

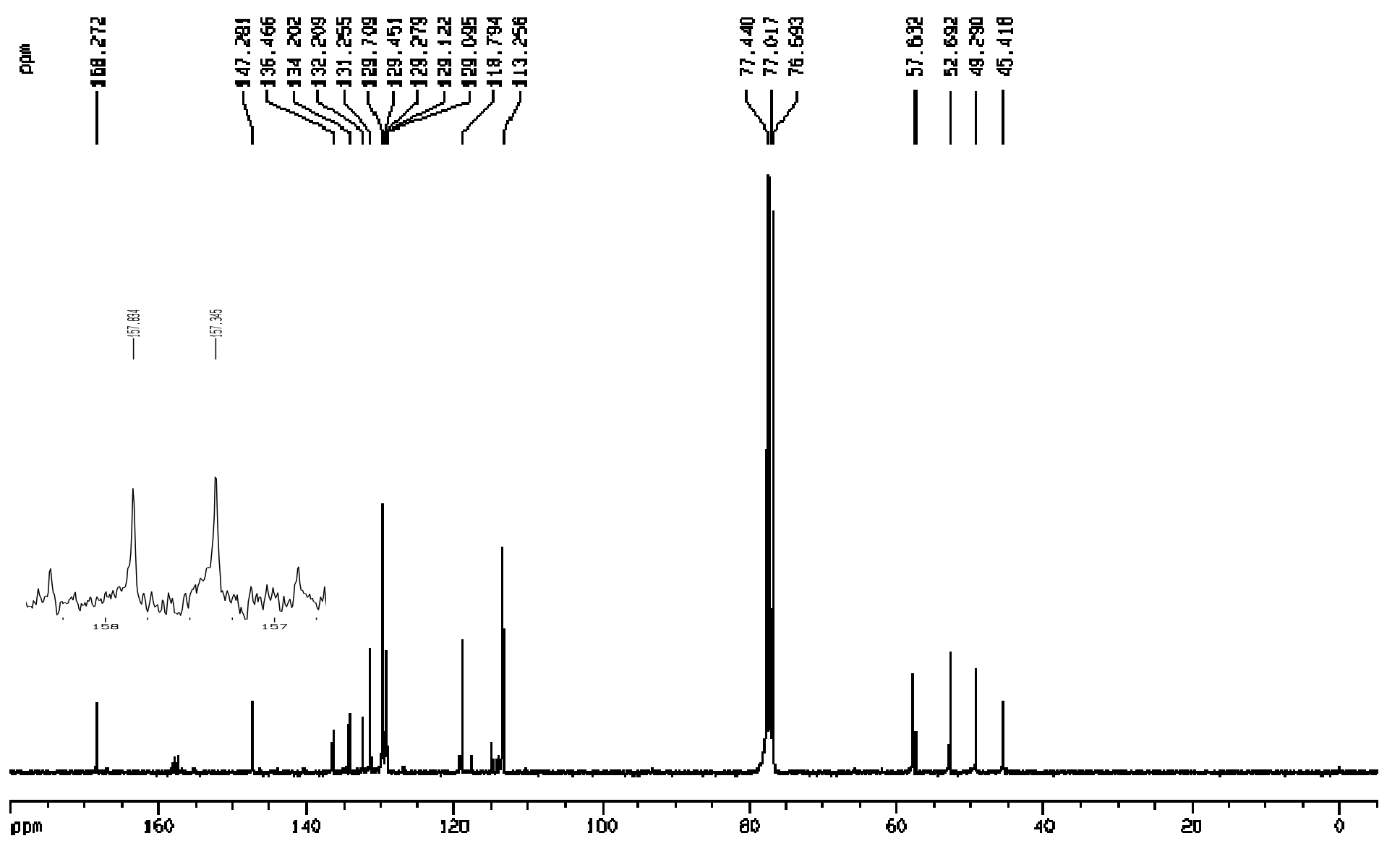

Currenn Dess Parbueters

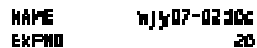

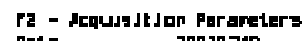

Date-
Ture

Ture

19.46

PULPPOG 2909:

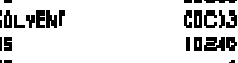

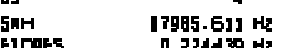

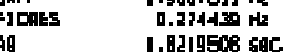

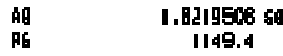

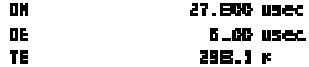

पI 2.0000000 ses

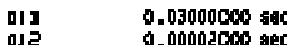

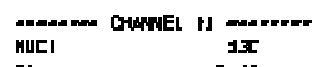

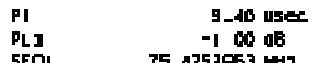

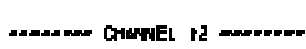

CPDPAG G MUILASB

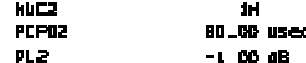

PLI2

Fror

Fi - Procesound paraneters

$5 \mathrm{~F} \quad 75,1577-180$

MUKE

II $\quad 1$

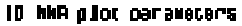

cx $2100 \mathrm{con}$

Fi
FIF

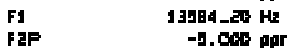

princh

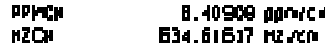


17- ${ }^{1} \mathrm{H}$ NMR

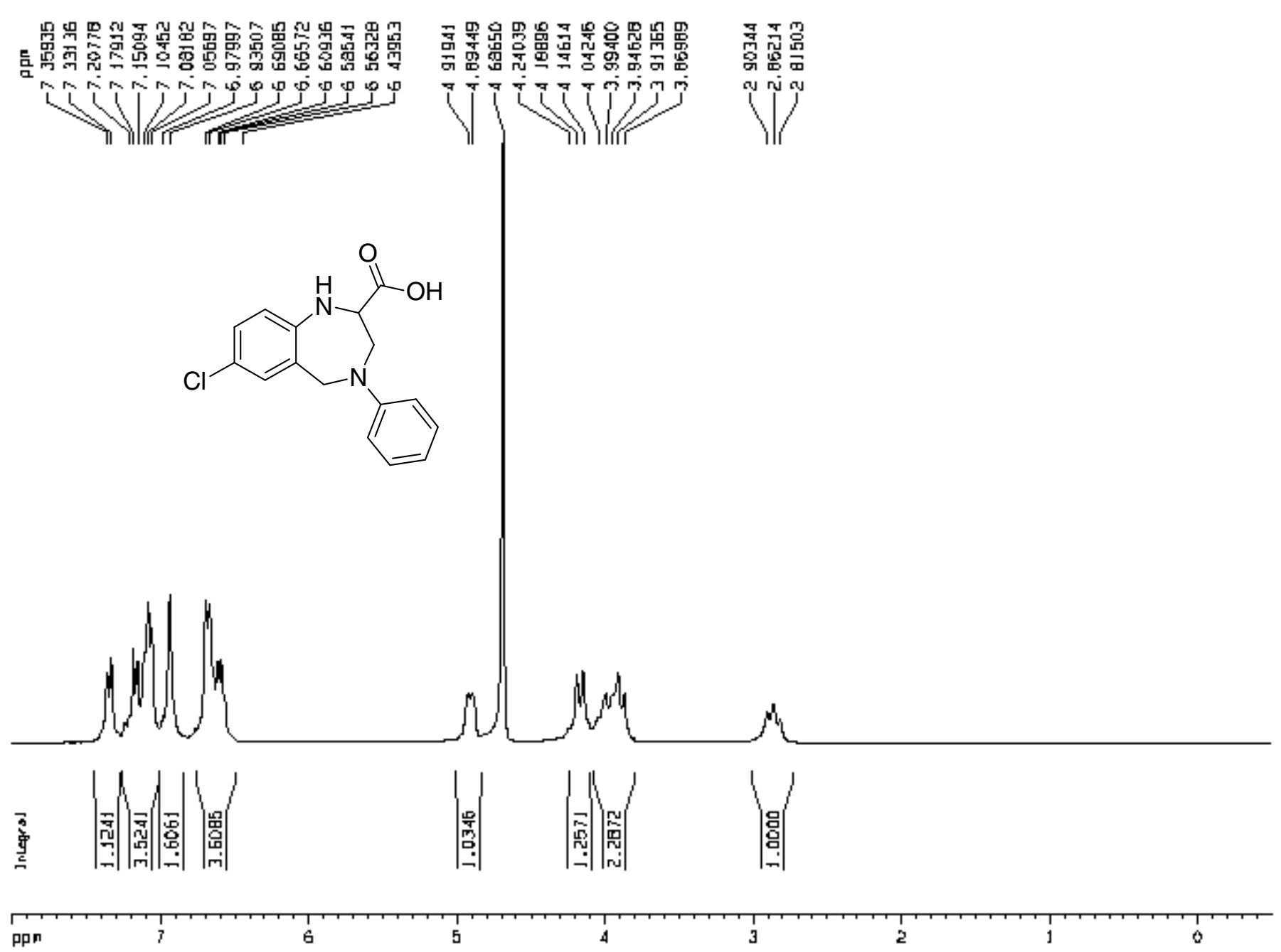

Gurcenc Doca Parangegr:

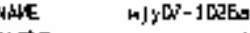

PPOONO

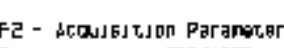

Dere_ a dorjoz

11, 17311

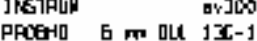

PUPA0

10

5ayte

4AE 15

$5 \mathrm{hH} \quad \mathrm{Heg} 806 \mathrm{HL}$

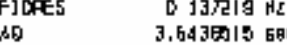

P6

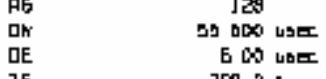

$5292 \mathrm{~s}$

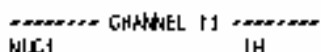

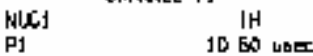

$-10 \mathrm{OB}$

Fe - Proceen Jng paraphigre

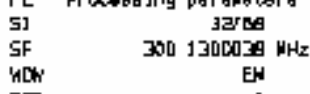

558

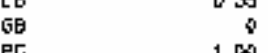

$10 \mathrm{WH}$ plat paranetera

$0 \quad 220 \mathrm{~cm}$

Cr $30 \mathrm{Con}$

FIP $\quad$ B.,00 pon

$\mathrm{FP}$

Fe -160 C6 Hs

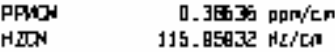

pp 
$17-{ }^{13} \mathrm{C}$ NMR
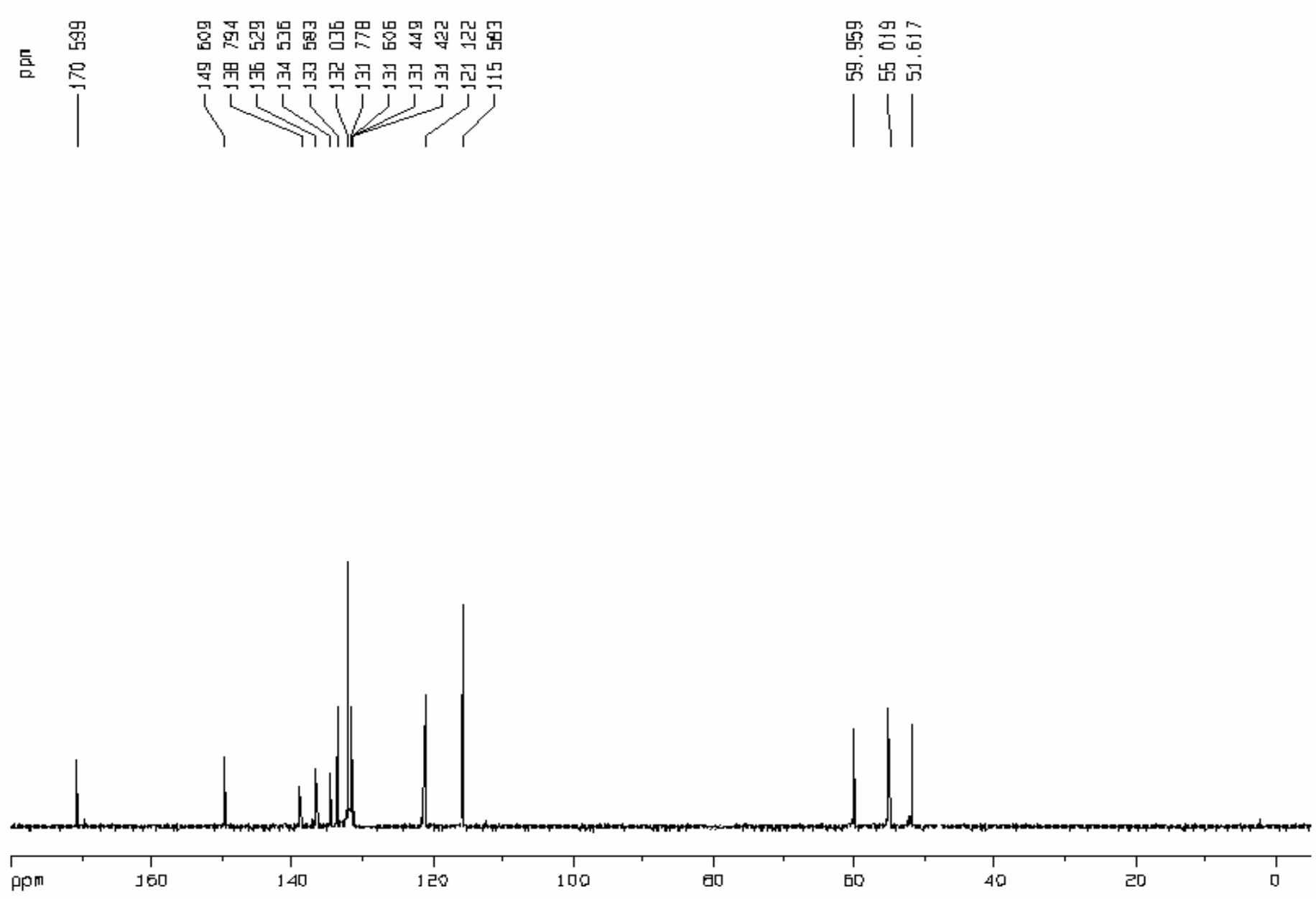

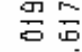

出的 
11e- ${ }^{1} \mathrm{H}$ NMR
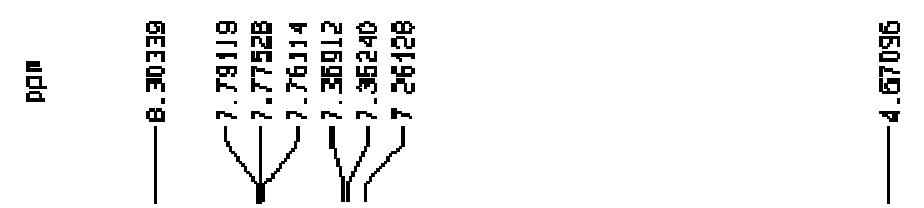

Current Dato Poroneters

MWE NyO7-093 TC

ExPHO

2 - Acquistkjon Paraneter

Dare_ 200I031

11.12

JKG7PMn क

PILPFog 5 m dul 13a-

TD
5O YEMT

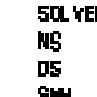

Sit

F JUPES O.JJT219 H2

10 3. EAJBEIG GEC

PG 406.4

DE

(c)

25. $\mathrm{K}$ K

5.000000 of 5ee

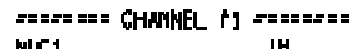

MUES

P) 9. 30 uset

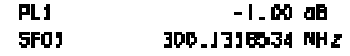

F2 - Procesajng paranecters

G] Procastung perangerar

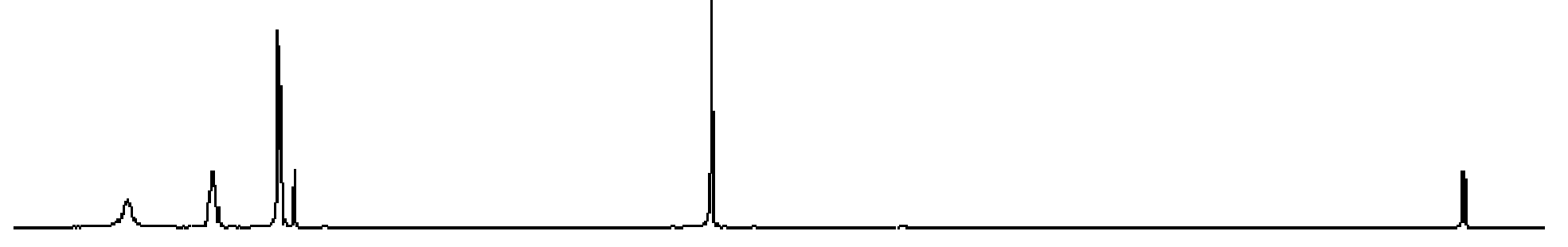

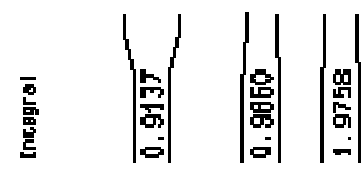

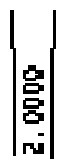

SFM 300 . J J00009 $\mathrm{HH}_{2}$

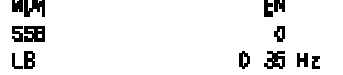

PC: 1

10 wim plot paranaters

C.

FyP

$\begin{array}{ll}\mathrm{FI} & 2701.13 \mathrm{HI} \\ \mathrm{FtP} & -0.504 \mathrm{FPn}\end{array}$

PFAlin $\quad-156-b \mathrm{HI}$

HILH IZM.EDI5g HI/CA 


\section{$11 \mathrm{e}-{ }^{13} \mathrm{C}$ NMR}
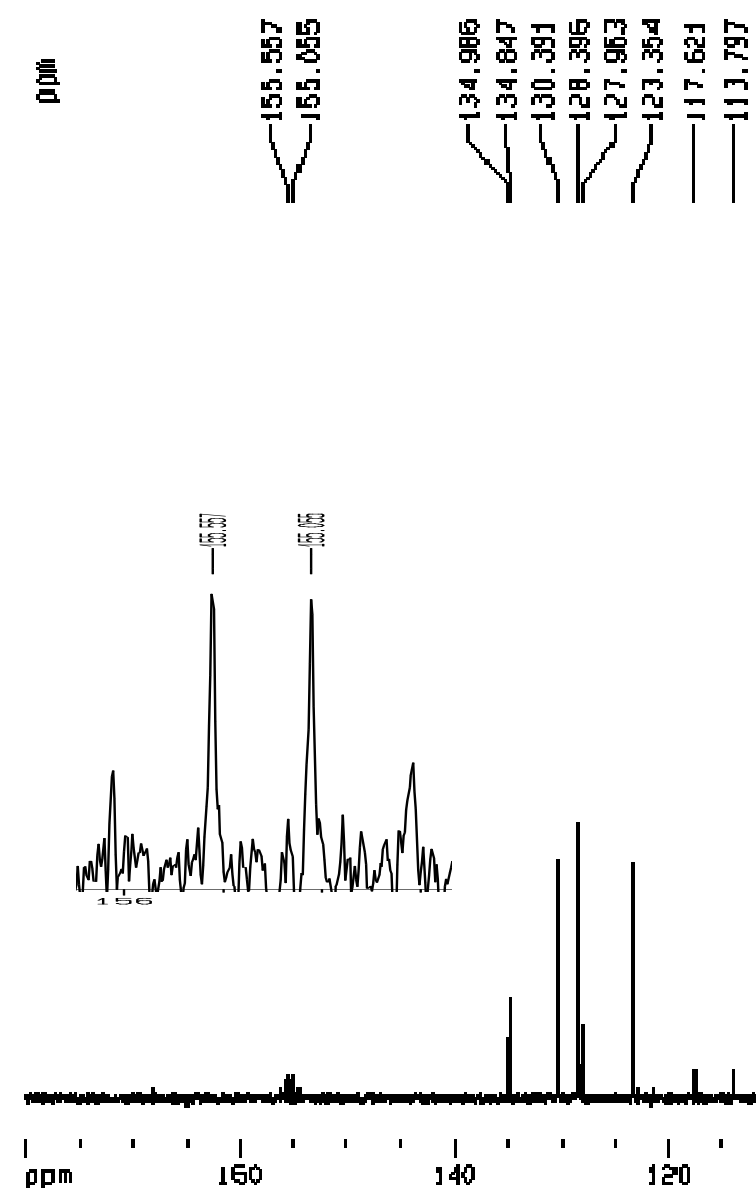

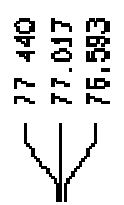

is

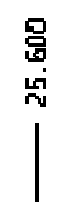

Curremt data Paranetere

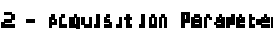

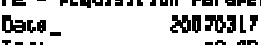

Indi

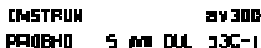

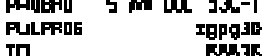

TI

SOLUE

DE

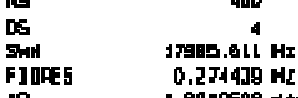

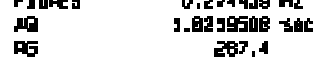

整

算

ad

dar

27.sor usec Gion $\mathrm{n}$.

$2.000000100 \mathrm{he}$

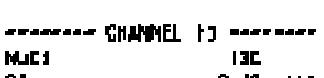

PJIJ I3C

RLI $\quad-1.00$. $0 \mathrm{~B}$

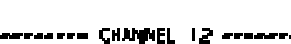

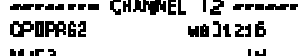

PULC $\quad$ IH

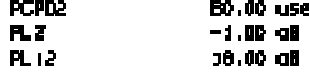

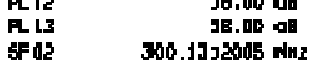

F2 - Processumin paraneters

FI - Processumy paranteters

51 $79.4577496 \mathrm{MHz}$

Le

PC

I0 Nan p Jat parameters

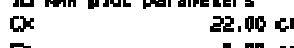

5

FIP
FJ
FEP
FE
PHH
HEC

$100.000 \mathrm{cph}$

-5.000 pp

$-371.34 \mathrm{~Hz}$

a. 46909 ppurch
$69.61517 \mathrm{mz} / \mathrm{CO}$ 
$13 a+14 a-{ }^{1} \mathrm{H}$ NMR

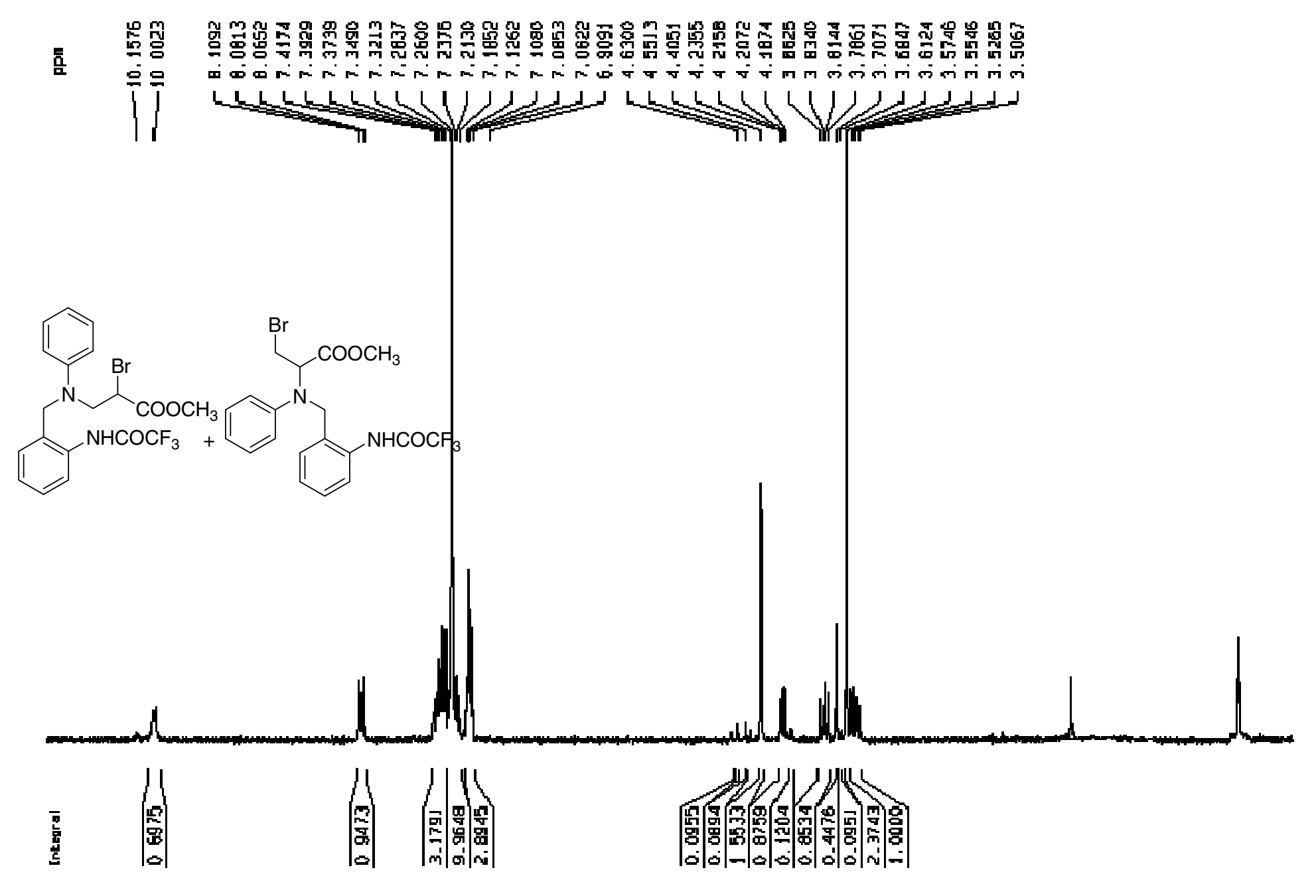

Gurrent Dot In Poraneters

MuE DIMDE-1J Jo

ExPAO

F 2 - MCquei luon Pardmeter

Dote

2016! 110

J5TPly

PFôH 5 un Du ixid

PII 30

2930
65536

SRVENT COCJ

HS

DS

5172,839

IDRES D. $194190 \mathrm{H}_{2}$

B.3016a0 set

ON as. DNO UEe

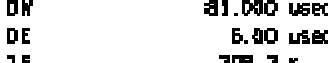

$1 E 2001290.3 \%$

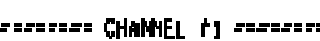

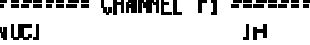

PI 990 une

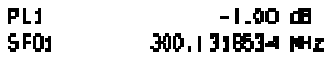

FZ - Procesf Jng parangters

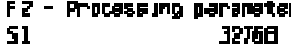

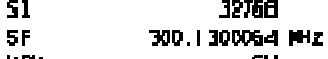

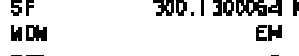

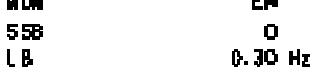

PE 1 ID

10 MNR plot porometers

Gh $22.00 \mathrm{~cm}$

$\begin{array}{ll}\text { GY } & 50.00 \mathrm{~cm} \\ \mathrm{FIP} & \mathrm{I1.000} \text { pan }\end{array}$

$\begin{array}{ll}\text { F IP } & 11.000 \mathrm{pan} \\ \mathrm{F} & 3300.43 \mathrm{HL}\end{array}$

F2 - 20005

FE
PFCH
HIOH

ה


$13 a+14 a-{ }^{-} C N M R$

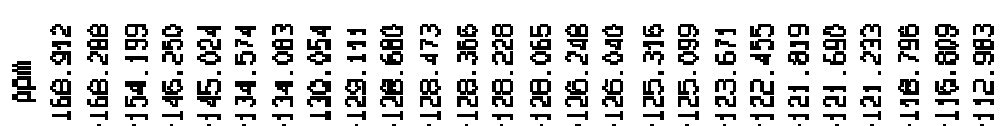

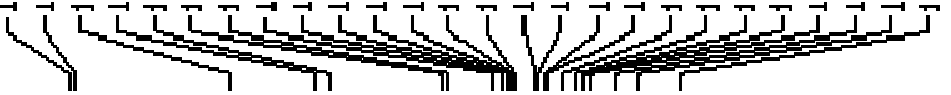
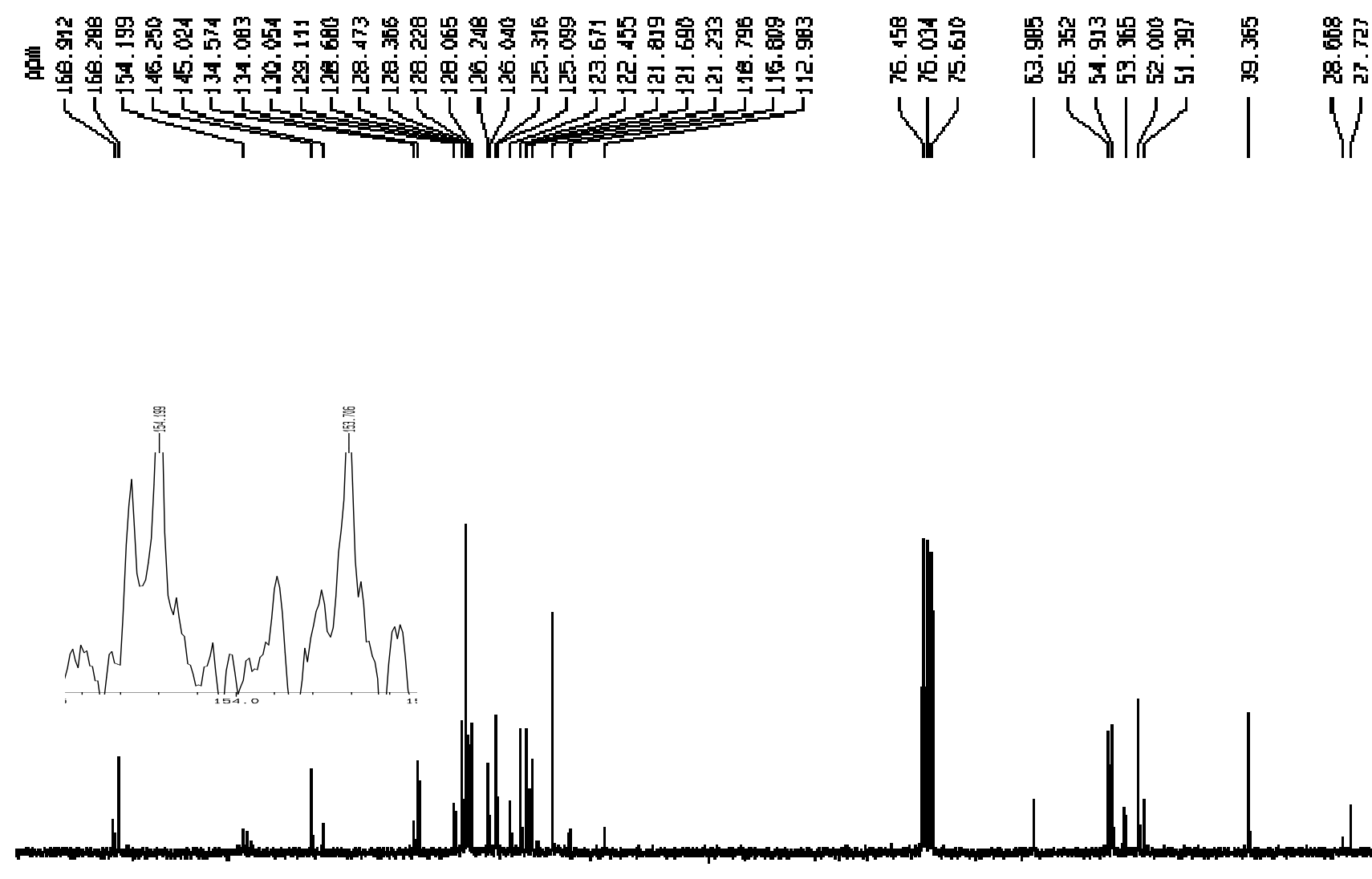

p pm

160

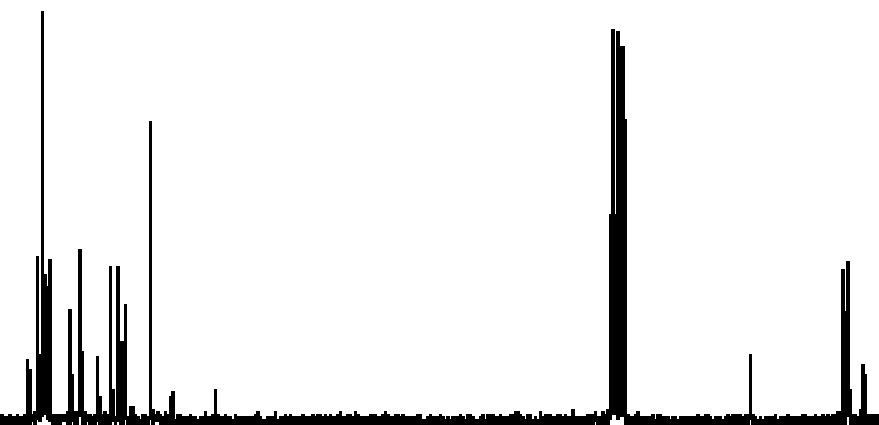

Ien
60
60
F2 - Moluusutjon Partieters

Tare-

PFiPAD $5 \mathrm{~m}$ all $135 \mathrm{C}$

To

SA YEM1

5WH TTMES. $514 \mathrm{~Hz}$

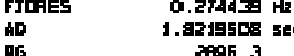

奛
OF
of

S.co unac
$299.6 \mathrm{~F}$

2.00000000 sec

0 a 00002000 sec

HNMEL H] -......

$\begin{array}{ll}\text { MucJ } & 10 \\ \text { PLJ } & 9.40\end{array}$

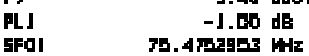

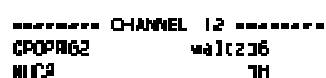

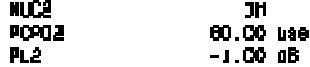

PLJ2 $\quad$ 18.50

PLOP

FE' - Processing parangrors
5I J276a

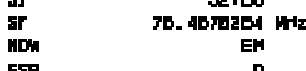

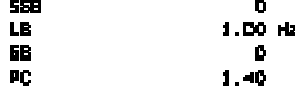

ID NHP plok parahterte

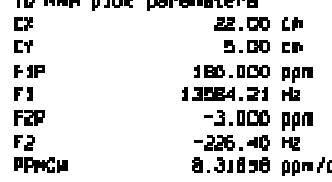

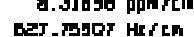




\section{X-ray structure of $15 \mathrm{e}$}

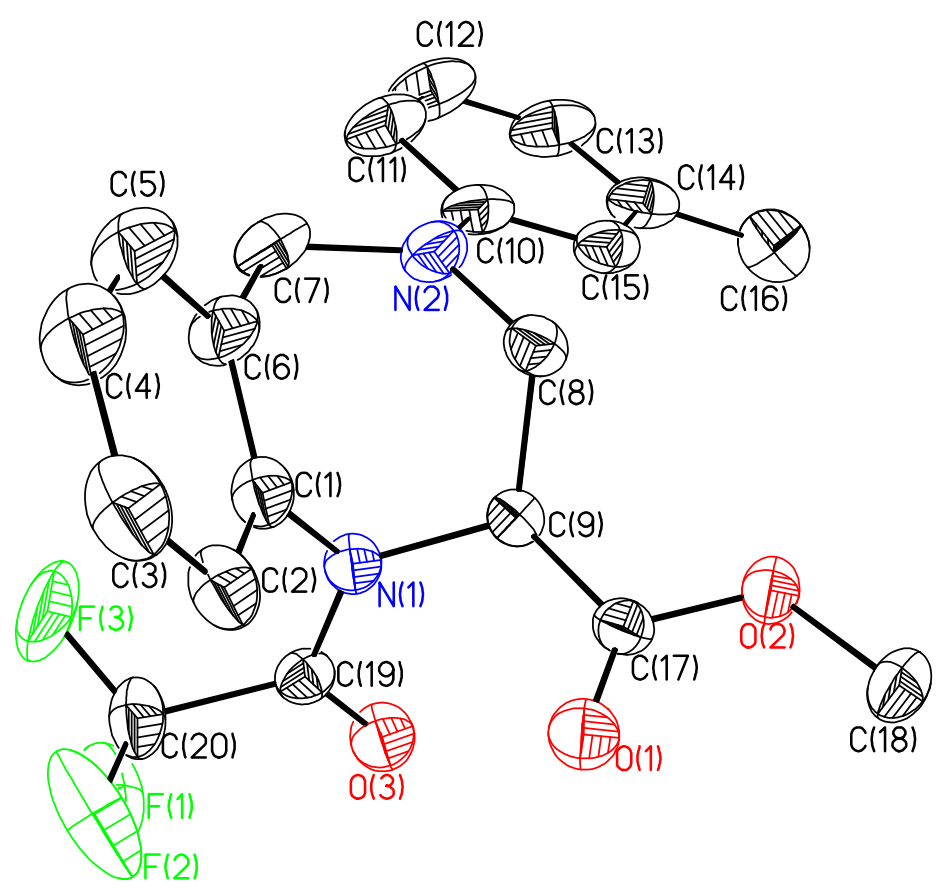

Figure S1. X-ray Single Crystal Structure of 15e (Hydrogen Atoms Are Omitted for Clarity)

\section{References.}

(1) Wright, S. W.; Dow, R. L.; McClure, L. D.; Hageman, D. L.; Tetrahedron Lett., 1996, 37, 6965-6968.

(2) Kumar, H. M. S.; Rao, M. S.; Chakravarthy, P. P.; Yadav, J. S. Tetrahedron: Asymmetry, 2004, 15, 127. 